\title{
Comprehensive Evaluation of the Absorption, Photophysical, Energy Transfer, Structural, and Theoretical Properties of $\alpha$-Oligothiophenes with One to Seven Rings
}

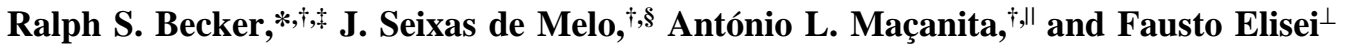 \\ Instituto de Tecnologia Química e Biológica, Rua da Quinta Grande n6, 2780 Oeiras, Portugal, Department \\ of Chemistry, University of Arkansas, Fayetteville, AR, Departamento de Química, Universidade de Coimbra, \\ 3000 Coimbra, Portugal, Departamento de Química do IST, Lisboa, Portugal, and Dipartimento di Chimica, \\ Universitá di Perugia, Perugia, Italy
}

Received: March 20, 1996; In Final Form: August 22, $1996^{\otimes}$

\begin{abstract}
A large basis set of $\alpha$-oligothiophenes with two to seven rings ( $\alpha 2-\alpha 7)$, also including thiophene, $\alpha 1$, have been investigated in five solvents regarding absorption, fluorescence and phosphorescence, quantum yields of fluorescence $\left(\phi_{\mathrm{F}}\right)$ and triplet formation $\left(\phi_{\mathrm{T}}\right)$, lifetimes of fluorescence and the triplet state, quantum yields of singlet oxygen production $\left(\phi_{\Delta}\right)$, all rate constants $k_{\mathrm{F}}, k_{\mathrm{IC}}, k_{\mathrm{ISC}}$, and several of the foregoing as a function of temperature. Ten different theoretical calculations across several levels including three levels of $a b$ initio have been carried out regarding which conformer is lowest in energy and the $\Delta H$ 's among all conformers of $\alpha 2, \alpha 3$ and $\alpha 5$, as well as calculations of transitions energies of the $\alpha$-oligothiophenes. We have shown that the (1) ${ }^{1} \mathrm{Bu}$ state is the lowest singlet state for all $\alpha 2-\alpha 7$ in any solvent, in contradiction to previous predictions for the higher members. Based on absorption and fluorescence data and calculations of atomic charges in $\mathrm{S}_{0}$ and $S_{1}$, the ground state is twisted while the excited state is planar (quinoidal-like). Significant charge transfer occurs between $S_{0}$ and $S_{1}$ but not $S_{0}$ and $T_{1}$. For all $\alpha 2-\alpha 7, \phi_{\text {IC }}$ is small, $k_{F}^{0}$ is approximately constant while $k_{\mathrm{ISC}}$ decreases significantly from $\alpha 2$ to $\alpha 7$. The decrease is $k_{\mathrm{ISC}}$ is believed to arise from a decrease in matrix elements of the type $\left\langle{ }^{1} \Psi_{\mathrm{CT}}\left|\mathrm{H}^{\prime}\right|^{3} \Psi_{1}\right\rangle$. The essential lack of phosphorescence is assigned as originating from inter-ring twisting mode coupling between $\mathrm{T}_{1}$ and $\mathrm{S}_{0}$. Triplet energy transfer to ${ }^{3} \mathrm{O}_{2}$ to produce ${ }^{1} \mathrm{O}_{2}$ is highly efficient for $\alpha 2-\alpha 5$. Based on all data, the first $\alpha$ n representative of $\alpha$-polythiophene is $\alpha 5$.
\end{abstract}

\section{Introduction}

The $\alpha$-oligothiophenes (designated $\alpha 1, \alpha 2, \alpha 3, \ldots$ where $n$ $=1,2,3, \ldots$, respectively)

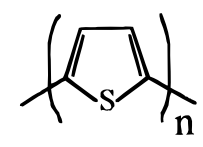

are currently of keen interest because (1) they are interesting analogues of polyenes, (2) they are good singlet oxygen sensitizers and biophotosensitizers, and (3) particularly those with higher $n$ are important for their use in nonlinear optics applications, charge storage, and molecular electronics.

There has been only limited information available on the fluorescence, fluorescence quantum yields $\left(\phi_{\mathrm{F}}\right)$ and lifetimes $\left(\tau_{\mathrm{F}}\right)$, triplet yields $\left(\phi_{\mathrm{T}}\right)$ and lifetimes $\left(\tau_{\mathrm{T}}\right)$, singlet oxygen yields $\left(\phi_{\Delta}\right)$, and the rate constants connecting the excited states and those connecting excited states to the ground state. Recently, ${ }^{1}$ all the above aspects except $\phi_{\Delta}$ were evaluated in one solvent. Also, $\alpha 2-\alpha 6$ have been studied ${ }^{2,3}$ relative to $\phi_{\mathrm{F}}$ and $\phi_{\mathrm{F}}$ for $\alpha 2-$ $\alpha 6$ and $\phi_{\Delta}$ for $\alpha 3-\alpha 6 .^{4}$ Other scattered data exist for some $\alpha n$ 's regarding some of the photophysical parameters. Some information exists for films, but again it is limited, even more than for solutions, in terms of the number of oligothiophenes and the photophysical parameters evaluated. One area receiving considerable attention is theoretical, expecially regarding torsional (inter-ring) barriers, $\Delta H$ of trans and cis conformers, and

\footnotetext{
$\dagger$ Instituto de Tecnologia Química e Biológica.

¥ University of Arkansas.

$\S$ Universidade de Coimbra.

"Departamento de Química do IST.

$\perp$ Universitá di Perugia.

${ }^{\otimes}$ Abstract published in Advance ACS Abstracts, November 1, 1996.
}

energy of states, but again, these have been limited essentially to $\alpha 2-\alpha 3$, particularly $\alpha 2$. We shall again consider all of the foregoing at the appropriate time.

One of the dominant questions of importance is the state order, particularly relating to the two lowest singlet $\pi, \pi^{*}$ states. The symmetry of the trans oligothiophenes varies depending upon the odd-even number of rings, $C_{2 V}$ for odd, $C_{2 h}$ for even. The states of interest are the ${ }^{1} \mathrm{Bu}$ and ${ }^{1} \mathrm{Ag}$ ones within $C_{2 h}$ symmetry, and this notation, or ${ }^{1} \mathrm{~B}$ and ${ }^{1} \mathrm{~A}$, is commonly carried through for $C_{2 v}$ symmetry. This order is considered important in terms of the relationship/identity of $\alpha$-oligothiophenes and polyenes with the same number of double bonds. For $\alpha 2$, in solution the $(1)^{1} \mathrm{Bu}$-like state is the lowest where the $(2)^{1} \mathrm{Ag}$ like state has been located above it by two-photon spectroscopy, ${ }^{5}$ and this $(2)^{1} \mathrm{Ag}$-like state also has been located above the $(1)^{1} \mathrm{Bu}-$ like state in a crystalline film of $\alpha 6^{6}$ (all state symbols should read "like", since the exact molecular symmetry and degree of state mixing is not known; however, we shall drop this addition for simplicity). Based on some spectroscopic data on $\alpha, \omega$ dithienylpolyenes, restricted CI (configuration interaction) calculations, extrapolated curves of ${ }^{1} \mathrm{Ag}$ and ${ }^{1} \mathrm{Bu}$ states energies of some polythiophene oligomers, and the difference in state energies expected for $\alpha 6$ in the crystalline state vs alkane solution, the $(2)^{1} \mathrm{Ag}$ state was assigned below the $(1)^{1} \mathrm{Bu}$ state for $\alpha 6$ and all other polythiophene oligomers with more than six rings $(\alpha 6, \alpha 7, \ldots.){ }^{7}$ We will show that this is not the case.

Other calculations ${ }^{8}$ predict the $(2)^{1} \mathrm{Ag}$ state already below the (1) ${ }^{1} \mathrm{Bu}$ state at $\alpha 2-\alpha 4$, with crossing occurring between $\alpha 4$ and $\alpha 5$. However, by restriction of the CI (which seems to have no real rational justification as was also done before),${ }^{7}$ the $(1)^{1} \mathrm{Bu}$ state was now predicted to be lowest for all $\alpha$ 's through $\alpha 6$, $\alpha 7$. Still other calculations ${ }^{9-11}$ on $\alpha 2$ and some higher $\alpha n^{\prime}$ 's in 
some cases give varying results regarding the relative ordering of the states for $\alpha 2$ and place the $(2)^{1} \mathrm{Ag}$ state lowest for $\alpha 3$ and $\alpha 4 .{ }^{9}$

We will show that despite any theoretical and/or comparative experimental data to the contrary, the $(1)^{1} \mathrm{Bu}$ state is the lowest excited singlet state in all $\alpha$-oligothiophenes from $\alpha 2$ to and including $\alpha 7$. We will also determine the following in five solvents for $\alpha 2-\alpha 7$ : (1) fluorescence $\left(\phi_{\mathrm{F}}\right)$ and triplet $\left(\phi_{\mathrm{T}}\right)$ quantum yields, $(2)$ fluorescence $\left(\tau_{\mathrm{F}}\right)$ and triplet $\left(\tau_{\mathrm{T}}\right)$ lifetimes, (3) all determinable rate constants $k_{\mathrm{F}}^{0}, k_{\mathrm{ISC}}$ (intersystem crossing), $k_{\mathrm{IC}}$ (internal conversion), and $k_{\mathrm{NR}}$ (nonradiative), (4) absorption (and $\epsilon$ values) and fluorescence spectra (and phosphorescence for $\alpha 1$ ) at 298 and $77 \mathrm{~K}$, (5) singlet oxygen quantum $\left(\phi_{\Delta}\right)$ yields of $\alpha 2-\alpha 5$ and $\alpha 7,(6) \tau_{\mathrm{F}}$ and $\phi_{\mathrm{F}}$ as a function of temperature for $\alpha 3$ and $\alpha 7$, (7) 10 different theoretical calculations of various levels for a broad variety of $\alpha n$ 's, including three levels of $a b$ initio that evaluate which conformer is the lowest in energy for all conformers of $\alpha 2, \alpha 3$, $\alpha 5,(8)$ calculations of atomic charges and their changes between $S_{0}$ and $S_{1}$ as well as $T_{1}$, (9) calculation of transition energies and oscillator strengths for $\alpha 1-\alpha 5$, and (10) correlation of absorption, emission, and photophysical parameters as a function of $n$ or $1 / n$ where $n$ is the number of rings. All these will be integrated to elucidate the behavior of the absorption and photophysical properties of $\alpha 1-\alpha 7$.

\section{Experimental Section}

Thiophene was purchased from Riedel de Häen and was used as received. Bithiophene and terthiophene were purchased from Aldrich and were respectively purified by sublimation and recrystallization. Tetrathiophene and dibutylheptathiophene were a kind gift from J. Kagan and H. Winberg, respectively. These were purified by column chromatography. Hexathiophene was provided by H. Naarmann (BASF).

Pentathiophene was synthesized by bromination of terthiophene in the $\alpha$ positions followed by reaction with 2-bromothiophene via Grignard coupling in the presence of a nickel catalyst $\left.\left(\mathrm{Ni}\left[\mathrm{C}_{6} \mathrm{H}_{5}\right)_{2} \mathrm{PCH}_{2} \mathrm{CH}_{2} \mathrm{P}\left(\mathrm{C}_{6} \mathrm{H}_{5}\right)_{2}\right] \mathrm{Cl}_{2}\right)$ synthesized as elsewhere reported (and dried with $\mathrm{SOCl}_{2}$ ). ${ }^{12}$ The final product, $\alpha 5$, was recrystallized from dichloromethane.

All the solvents were of spectroscopic or equivalent grade, except for methylcyclohexane (which was purified over a mixture of $\mathrm{H}_{2} \mathrm{SO}_{4} / \mathrm{HNO}_{3}$, then distilled, and finally chromatographed on an $\mathrm{Al}_{2} \mathrm{O}_{3} / \mathrm{SiO}_{4}$ column) and ethanol (dried and purified by distillation over $\mathrm{CaO}$ ). All the other solvents were used without further purification. The solutions used $\left(1 \times 10^{-5}\right.$ to $10^{-6} \mathrm{M}$ ) were deoxygenated by either $\mathrm{N}_{2}$ or Ar bubbling. Anthracene, naphthalene (J. K. Baker Chemical Co.), and benzophenone (Aldrich Chemical Co.) were used without further purification.

Absorption and fluorescence spectra were run with a Beckman DU-70 and a SPEX Fluorolog spectrometer, respectively. All the fluorescence spectra were corrected for the wavelength response of the system.

The fluorescence quantum yields at $293 \mathrm{~K}$ were measured using several standards, namely, methyl 1-pyrenoate $\left(\phi_{\mathrm{F}}=0.83\right.$ in cyclohexane) and 3-chloro-7-methoxy-4-methylcoumarin $\left(\phi_{\mathrm{F}}\right.$ $=0.12$ in cyclohexane $\left.{ }^{13}\right)$. Terthiophene in ethanol $\left(\phi_{\mathrm{F}}=0.054\right)$ was also used for internal verification of the obtained values. The fluorescence quantum yields at $77 \mathrm{~K}$ were obtained by running under the same experimental conditions the solution done at $293 \mathrm{~K}$, avoiding by this way external interferences. The $\phi_{\mathrm{F}}$ value was than obtained by assuming a $20 \%$ "shrinkage" of the ethanol solvent on going from 293 to $77 \mathrm{~K}$.
The molar extinction coefficients $(\epsilon)$ were obtained with seven solutions of different concentrations. The slope of the plot of the absorption values (at the maximum wavelength of absorption) vs the concentration values gave us the $\epsilon$ values with correlation values $\geq 0.999$.

The radiative rate constant $k_{\mathrm{R}}$ was calculated with the Strickler-Berg ${ }^{14}$ equation

$$
k_{\mathrm{R}}^{\mathrm{SB}}=\left(2.88 \times 10^{-9}\right) n^{2}\left\langle\bar{\nu}_{\mathrm{F}}^{-3}\right\rangle_{\mathrm{AV}^{-1}} \int \frac{\epsilon(\bar{v})}{\bar{v}} \mathrm{~d} \bar{v}
$$

with

$$
\left\langle\bar{v}_{\mathrm{F}}^{-3}\right\rangle_{\mathrm{AV}^{-1}}=\frac{\int I(\bar{v}) \mathrm{d} \bar{v}_{\mathrm{F}}}{\int \bar{v}_{\mathrm{F}}^{-3} I\left(\bar{v}_{\mathrm{F}}\right) \mathrm{d} \bar{v}_{\mathrm{F}}}
$$

using the integrated first absorption band and the fluorescence band. For thiophene, since there is no fluorescence, the use of the equation of Förster ${ }^{15}$ was needed:

$$
k_{\mathrm{R}}^{\mathrm{F}}=\left(2.88 \times 10^{-9}\right) n^{2} \int \frac{\epsilon(\bar{v})\left(2 \bar{v}_{0}-\bar{v}\right)^{3}}{\bar{v}} \mathrm{~d} \bar{v}
$$

Fluorescence decays were obtained using a time-correlated single-photon-timing technique with a home-built apparatus as previously described ${ }^{16}$ except for the wavelength shift, which is now $300 \mathrm{fs} / \mathrm{nm}$. The obtained fluorescence decays were deconvoluted in a Microvax 3100 employing the method of modulating functions. ${ }^{17}$

The experimental setup used for triplet spectra and triplet yields of $\alpha 2-\alpha 5$ was that described elsewhere. ${ }^{18,19}$ Absorption spectra were recorded every $10 \mathrm{~nm}$ averaging at least 5 shots per wavelength recorded. The triplet-triplet molar extinction coefficients $\left(\epsilon_{\mathrm{T}}\right)$ were evaluated using the energy transfer method from naphthalene (in benzene, dioxane, and ethanol) and from benzophenone (in benzene and acetonitrile) to $\alpha n$ 's. A solution of the sensitizer, $\mathrm{S}$, had an absorbance of 1.5-1.6 and the $\alpha$ n had an absorbance of $0.15-0.25$ at the excitation wavelengths of 308 and $347 \mathrm{~nm}$ for naphthalene and benzophenone, respectively.

The equation ${ }^{20}$

$$
\epsilon_{\mathrm{T}}(\mathrm{A})=\epsilon_{\mathrm{T}}(\mathrm{S}) \frac{\Delta A_{\mathrm{A}}-\Delta A_{\mathrm{dir}}}{\Delta A_{\mathrm{S}} f_{\mathrm{S}}}\left\{\frac{k_{2}-k_{1}}{k_{2}} \exp \left[-\frac{\ln \left(k_{2} / k_{3}\right)}{\left(k_{2} / k_{3}\right)-1}\right]\right\}^{-1}
$$

was used where the acceptor $\mathrm{A}=\alpha n$ and $\mathrm{S}$ is the sensitizer. This equation takes into account the following: $\Delta A_{\mathrm{S}}$, the absorbance change of sensitizer alone; $\Delta A_{\mathrm{A}}$, the absorbance change of the maximum; $k_{2}$, the decay rate constant of the sensitizer in the presence of the acceptor; $k_{3}$, the decay rate constant of the sensitized triplet of the acceptor; $\Delta A_{\text {dir }}$, the direct excitation of the acceptor; $f_{\mathrm{S}}$, the fraction of light absorbed by the sensitizer in the solution of $\mathrm{S}+\mathrm{A}$ with respect to the sensitizer alone. Triplet properties of the sensitizers in some solvents were taken from the literature ${ }^{21,22}\left(\epsilon_{\mathrm{T}}\right.$ in $\left.\mathrm{M}^{-1} \mathrm{~cm}^{-1}\right)$ : for naphthalene (benzene), $\epsilon_{\mathrm{T}}=13200(420 \mathrm{~nm}), \phi_{\mathrm{T}}=0.75$; for naphthalene (ethanol), $\epsilon_{\mathrm{T}}=40000(420 \mathrm{~nm})$; for benzophenone (benzene), $\epsilon_{\mathrm{T}}=7200(530 \mathrm{~nm}), \phi_{\mathrm{T}}=1.0$; for benzophenone (acetonitrile), $\epsilon_{\mathrm{T}}=6500(530 \mathrm{~nm}), \phi_{\mathrm{T}}=1.0$. The $\epsilon_{\mathrm{T}}$ values of anthracene and naphthalene in dioxane were determined in this work. The $\phi_{\mathrm{T}}$ of anthracene was determined by the heavy atom effect of 4-bromo- $N, N^{\prime}$-dimethylaniline on the fluorescence quantum yield and triplet population. ${ }^{19,23}$ Energy transfer between naphthalene (donor) and anthracene (acceptor) were used to obtain $\epsilon_{\mathrm{T}}$ of naphathalene in dioxane: for 
TABLE 1: Photophysical and $\phi_{\Delta}$ Data for $\alpha$-Oligothiophenes in Benzene ${ }^{a, b}$

\begin{tabular}{cccccccccc}
\hline cpd & $\phi_{\mathrm{F}}$ & $\tau_{\mathrm{F}}(\mathrm{ns})$ & $k_{\mathrm{F}}^{0}$ & $k_{\mathrm{NR}}$ & $\phi_{\mathrm{T}}$ & $\phi_{\Delta}$ & $k_{\mathrm{IC}}$ & $k_{\mathrm{ISC}}$ & $\tau_{\mathrm{T}}(\mu \mathrm{s})$ \\
\hline$\alpha 2$ & 0.026 & 0.046 & 0.55 & $21^{e}$ & 0.99 & 0.96 & $0.11^{c}$ & 22 \\
$\alpha 3$ & 0.07 & 0.16 & 0.44 & $5.8^{e}$ & 0.95 & $0.81^{d}$ & $0.03^{c}$ & 5.9 & 104 \\
$\alpha 4$ & 0.18 & 0.44 & 0.41 & 1.9 & 0.73 & 0.72 & 0.20 & 1.7 \\
$\alpha 5$ & 0.34 & 0.82 & 0.41 & 0.81 & 0.59 & 0.56 & 0.098 & 0.72 \\
$\alpha 6$ & 0.44 & 0.97 & 0.45 & 0.58 & & $\sim 0.36^{f}$ & 24 & \\
$\alpha 7$ & 0.36 & 0.82 & 0.43 & 0.79 & $\leq 0.6$ & $h$ & 0.049 & 0.73
\end{tabular}

${ }^{a}$ Solutions degassed with nitrogen or argon by bubbling. $k$ 's are $10^{9} \mathrm{~s}^{-1}$, and $\alpha 7$ is the di- $n$-butyl-substituted $\alpha 7$. ${ }^{b}$ Also see Table 2 , acetonitrile. ${ }^{c}$ Assumes $\left(1-\phi_{\mathrm{T}}-\phi_{\mathrm{F}}\right)=0.005 .{ }^{d}$ The value of $\phi_{\Delta}$ ranged from 0.65 to 0.85 depending on the technique used (see Experimental Section for techniques). This value was that from measuring emission of ${ }^{1} \mathrm{O}_{2}$. In acetonitrile a value of 0.74 was obtained by measuring ${ }^{1} \mathrm{O}_{2}$ emission. ${ }^{e} k_{\mathrm{NR}}$ obviously cannot be less than $k_{\mathrm{ISC}}+k_{\mathrm{IC}}$, but recall that errors in $\phi_{\mathrm{T}}$ for large $\phi_{\mathrm{T}}$ particularly effect the accuracy of $k_{\mathrm{IC}}$; see text. ${ }^{f}$ Reference 4 . We were unable to obtain sufficient concentration in benzene or dioxane to obtain a value using our techniques (see Experimental Section for techniques). ${ }^{g}$ Wintengs, V.; Valat, P.; Garnier, F. J. Phys. Chem. 1994, 98, 228 indicate $24 \mu$ s in dichloromethane and also $\alpha 4=35 \mu$ s. ${ }^{h}$ We have determined a tentative value of 0.25 in dioxane.

naphthalene (dioxane), $\epsilon_{\mathrm{T}}=15000(420 \mathrm{~nm})$; for anthracene (dioxane), $\epsilon_{\mathrm{T}}=50000(430 \mathrm{~nm}), \phi_{\mathrm{T}}=0.62$.

The product $\epsilon_{\mathrm{T}} \phi_{\mathrm{T}}$ for each $\alpha$ was obtained by the laser energy effect on the change of absorbance of an $\alpha$ measured at the $\lambda_{\max }$ of optically matched solutions $(A \approx 0.08)$ compared with benzophenone in acetonitrile and naphthalene in benzene as references/standards. Plots of $\Delta A$ vs laser dose were linear and passed through zero, indicating that only one-photon processes were occurring. Moreover, there was only a $<5 \%$ difference between $\epsilon_{\mathrm{T}} \phi_{\mathrm{T}}$ calculated with the two different references/standards. The triplet yield of $\alpha n$ 's was then calculated from the $\epsilon_{\mathrm{T}} \phi_{\mathrm{T}} / \epsilon_{\mathrm{T}}$ ratio.

For $\alpha 7$, the spectra and triplet parameters were obtained using the third harmonic of a Q-switched Nd:YAG laser where spectra and $\Delta A$ changes were obtained. The $\epsilon_{\mathrm{T}}$ was obtained by the partial depletion technique ${ }^{21}$ using benzophenone in benzene as a reference (actinometer) to measure $\phi_{\mathrm{T}}(\epsilon=7200$ at 530 $\left.\mathrm{nm}, \phi_{\mathrm{T}}=1^{22,24}\right)$. When the triplet yield was measured, special care was taken to have optically matched dilute solutions $(A \approx$ 0.2 in a $10 \mathrm{~mm}$ cell) and a low laser energy $(\leq 1.8 \mathrm{~mJ})$ to avoid multiphoton and $\mathrm{T}-\mathrm{T}$ annihilation effects. The region monitored for $\alpha 7$ was at the depletion minimum of $450 \mathrm{~nm}( \pm 10$ $\mathrm{nm}$ ), which appeared to be outside the $\mathrm{T}-\mathrm{T}$ absorption. Nonetheless, the $\phi_{\mathrm{T}}$ obtained should be an upper limit, since, if the triplet absorbs here, the $\epsilon_{\mathrm{T}}$ obtained will be too small. In our case a value of $87000 \mathrm{M}^{-1} \mathrm{~cm}^{-1}( \pm 15 \%)$ was obtained. The $\phi_{\mathrm{T}}$ was $0.60 \pm 15 \%$.

The singlet oxygen yields $\left(\phi_{\Delta}\right)$ of $\alpha n$ 's were determined by measuring the emission of ${ }^{1} \mathrm{O}_{2}$ in air-equilibrated benzene and acetonitrile solutions with a germanium diode detector. The luminescence emerging from the cuvette was passed through a filter combination (Glenn-Creston, cutoff $1050 \mathrm{~nm}$, and Kodak Wratten $87 \mathrm{c}$ gelatin filter, cutoff $870 \mathrm{~nm}$ ) and collected by a germanium diode (Judson J16 85p, $5 \mathrm{~mm}$ diameter). The detection system was at right angles to the excitation beam. After amplification with a homemade amplifier $(100 \mathrm{MHz}, 14$ $\mathrm{dB})$, the output was fed into a Tektronix DSA 602 digital sampling analyzer. The amplified signal extrapolated to zero time (in $\mathrm{mV}$ ) was plotted as a function of laser dose and compared with that of a reference, phenalenone, where $\phi_{\Delta}$ is 0.98 .25 The $\phi_{\Delta}$ of the $\alpha n$ 's was obtained from a ratio of the slopes and the known $\phi_{\Delta}$ of phenalenone.

\section{Results}

Table 1 presents comprehensive photophysical data of $\alpha 1-$ $\alpha 7$ (di- $n$-butyl), including singlet oxygen quantum yields $\left(\phi_{\Delta}\right)$ in benzene. Table 2 contains much of the same photophysical data, except for some $\phi_{\Delta}$ and $\phi_{\mathrm{T}}$ values, with four additional solvents. In most cases $\alpha 6$ could not be included because of its limited solubility. Table 3 presents data regarding absorption spectra and extinction coefficients, fluorescence and $\mathrm{T}-\mathrm{T}$ maxima, as well as the experimental $k_{\mathrm{F}}^{0}$ and $\mathrm{k}_{\mathrm{F}}^{0}$ from the Strickler-Berg equation (SB). ${ }^{14}$ Other tables will be considered in the Results and Discussion sections.

Absorption and Emission. Dependence on Solvent and Temperature. The solution absorption spectra at room temperature in any solvent for $\alpha 2-\alpha 7$ are generally devoid of structure (Figure 1). Table 3 gives representative absorption spectral data in dioxane. There is a relatively small effect of solvent on these maxima. However, the low-temperature absorption, as represented by the fluorescence excitation spectra, can have significant structure (Figure 2 and ref 1), particularly for $\alpha 2$ and $\alpha 3$. Moreover, there is a considerable red shift, $\sim 1600 \mathrm{~cm}^{-1}$, in the maxima. There is an excellent linear correlation between $1 / n$ and the maximum of the first transition, as well as the $0-0$ energy particularly for $\alpha 4-\alpha 7$ (Figure 3 ). If $\alpha 2$ and $\alpha 3$ are included, these points scatter somewhat compared to the others and change the slope slightly. Nonetheless, the linearity is good. This observation regarding the deviation of $\alpha 2$ in particular occurs for other kinds of relationships involving $1 / n$ (or $1 / N$ where $N$ is the length of the molecule in $\AA$ ) such as that for the maximum of the $\mathrm{T}-\mathrm{T}$ absorption, among others (Figure 3). These types of data plus photophysical results indicate that $\alpha 2$, and even $\alpha 3$, are not yet legitimate representatives of a polythiophene and that such a representation seemingly begins with $\alpha 4$. Actually, additional consideration of all the photophysical data indicates that the first true representative of a polythiophene is $\alpha 5$.

On the basis of the plot of $1 / n$ vs absorption maxima of $\alpha 4-$ $\alpha 7$ (corrected for the presence of two $n$-butyl groups), we can predict the expected maxima for $\alpha 8-\alpha 13$ and $\alpha \infty$ : $\alpha 8,456$ $\mathrm{nm} ; \alpha 9,466 \mathrm{~nm} ; \alpha 10,473 \mathrm{~nm} ; \alpha 11,480 \mathrm{~nm} ; \alpha 12,485 \mathrm{~nm}$; $\alpha 13,489 \mathrm{~nm} ; \alpha \infty, 555 \mathrm{~nm}$. The latter value for the absorption maximum of $\alpha \infty$ is quite insensitive to a change in the number of rings down to $50\left(\lambda_{\max }=538 \mathrm{~nm}\right), 40\left(\lambda_{\max }=535 \mathrm{~nm}\right)$, or even $30\left(\lambda_{\max }=526 \mathrm{~nm}\right)$, any one of which numbers may be a realistic value for the number of thiophene units in a real polythiophene

Figure 4 shows plots of $\epsilon$ vs $n$ and $1 / n$ for $\alpha 1-\alpha 7$. If you plot $\epsilon$ values for $\alpha 3-\alpha 5$ vs $1 / n$ and $n$, both plots are very linear. If you then use these to predict the extinction coefficients of $\alpha 6$ and $\alpha 7$ by extrapolation, the results are excellent from the $1 / n$ plot ( $\epsilon$ of $\alpha 6$ predicted to be 47400 compared to the experimental $47910 ; \epsilon$ of $\alpha 7$ predicted to be 50800 compared to the experimental 50470 ). On the other hand, extrapolation of the $n$ (vs $\epsilon$ ) plot gives 63400 for $\alpha 7$ vs 50470 found. This is a warning that even though there may be a linear relationship over a restricted basis set, it may in fact not permit any accurate predictive capability for other members of higher or lower $\alpha$. We will see later that this is true for other kinds of considerations 
TABLE 2: Photophysical Properties of $\alpha$-Oligothiophenes for $n=1-7$ in Various Solvents ${ }^{a} 1$

\begin{tabular}{|c|c|c|c|c|c|c|c|c|c|}
\hline \multicolumn{10}{|c|}{ Methylcyclohexane } \\
\hline \multicolumn{2}{|r|}{ cpd } & \multicolumn{2}{|l|}{$\phi_{\mathrm{F}}$} & \multicolumn{2}{|c|}{$\tau_{\mathrm{F}}(\mathrm{ns})$} & \multicolumn{2}{|r|}{$k_{\mathrm{F}}^{0}$} & \multicolumn{2}{|c|}{$k_{\mathrm{NR}}$} \\
\hline \multicolumn{2}{|r|}{$\alpha 2$} & \multicolumn{2}{|l|}{0.014} & \multicolumn{2}{|c|}{$\leq 0.1$} & \multicolumn{2}{|c|}{$\geq 0.14$} & \multicolumn{2}{|c|}{$\geq 9.9$} \\
\hline \multicolumn{2}{|r|}{$\alpha 3$} & \multicolumn{2}{|l|}{0.057} & \multicolumn{2}{|c|}{0.19} & \multicolumn{2}{|r|}{0.30} & \multicolumn{2}{|c|}{5.0} \\
\hline \multirow{2}{*}{\multicolumn{2}{|c|}{$\alpha 4$}} & \multicolumn{2}{|l|}{0.18} & \multicolumn{2}{|c|}{0.49} & \multicolumn{2}{|r|}{0.37} & \multicolumn{2}{|c|}{1.7} \\
\hline & $\alpha 5$ & 0.33 & & \multirow{2}{*}{\multicolumn{2}{|c|}{$\begin{array}{l}0.83 \\
0.76\end{array}$}} & \multirow{2}{*}{\multicolumn{2}{|c|}{$\begin{array}{l}0.39 \\
0.42\end{array}$}} & \multirow{2}{*}{\multicolumn{2}{|c|}{$\begin{array}{l}0.81 \\
0.90\end{array}$}} \\
\hline \multicolumn{2}{|r|}{$\alpha 7$} & \multicolumn{2}{|l|}{0.32} & & & & & & \\
\hline \multicolumn{10}{|c|}{ Ethanol } \\
\hline cpd & $\phi_{\mathrm{F}}$ & $\phi_{\mathrm{F}}(77 \mathrm{~K})$ & $\tau_{\mathrm{F}}(\mathrm{ns})$ & $k_{\mathrm{F}}^{0}$ & $k_{\mathrm{NR}}$ & $\phi_{\mathrm{T}}$ & $k_{\mathrm{IC}}$ & $k_{\mathrm{ISC}}$ & $\tau_{\mathrm{T}}(\mu \mathrm{s})$ \\
\hline$\alpha 2$ & 0.014 & 0.04 & $0.046^{b}$ & 0.35 & $\sim 21$ & 1.0 & 0.10 & $\sim 21$ & 100 \\
\hline$\alpha 3$ & 0.054 & 0.12 & 0.19 & 0.28 & 5.0 & 0.96 & $0.03^{c}$ & 5.1 & 91 \\
\hline$\alpha 4$ & 0.18 & 0.18 & 0.52 & 0.34 & 1.6 & 0.70 & 0.23 & 1.3 & 43 \\
\hline$\alpha 5$ & 0.32 & 0.34 & 0.88 & 0.36 & 0.77 & & & & 20 \\
\hline$\alpha 7$ & 0.29 & 0.28 & 0.82 & 0.35 & 0.87 & $\leq 0.6^{b}$ & 0.13 & 0.73 & \\
\hline \multicolumn{10}{|c|}{ Acetonitrile } \\
\hline cpd & $\phi_{\mathrm{F}}$ & $\tau_{\mathrm{F}}(\mathrm{ns})$ & $k_{\mathrm{F}}^{0}$ & $k_{\mathrm{NR}}$ & $\phi_{\mathrm{T}}$ & $\phi_{\Delta}$ & $k_{\mathrm{IC}}$ & $k_{\mathrm{ISC}}$ & $\tau_{\mathrm{T}}(\mu \mathrm{s})$ \\
\hline$\alpha 2$ & 0.013 & $\leq 0.1$ & $\geq 0.133$ & $\geq 10.1$ & 0.93 & 0.98 & $\geq 0.6$ & $\geq 9.5$ & 124 \\
\hline$\alpha 3$ & 0.056 & 0.18 & 0.311 & 5.1 & 0.90 & 0.74 & 0.24 & 5.0 & 62 \\
\hline$\alpha 4$ & 0.16 & 0.48 & 0.33 & 1.8 & 0.71 & 0.72 & 0.27 & 1.5 & 40 \\
\hline$\alpha 5$ & 0.33 & 0.90 & 0.37 & 0.74 & 0.63 & 0.50 & 0.011 & 0.70 & 20 \\
\hline$\alpha 7$ & 0.30 & 0.90 & 0.33 & 0.78 & $\leq 0.6^{b}$ & & 0.011 & 0.67 & \\
\hline \multicolumn{10}{|c|}{ Dioxane } \\
\hline cpd & $\phi_{\mathrm{F}}$ & $\tau_{\mathrm{F}}(\mathrm{ns})$ & $k_{\mathrm{F}}^{0}$ & & & $\phi_{\mathrm{T}}$ & $k_{\mathrm{IC}}$ & $k_{\mathrm{ISC}}$ & $\tau_{\mathrm{T}}(\mu \mathrm{s})$ \\
\hline$\alpha 2$ & 0.017 & $0.046^{b}$ & 0.37 & & & 0.94 & 0.93 & 20 & 146 \\
\hline$\alpha 3$ & 0.066 & 0.21 & 0.31 & & & 0.93 & 0.019 & 4.4 & 108 \\
\hline$\alpha 4$ & 0.18 & 0.49 & 0.36 & & & 0.67 & 0.31 & 1.4 & 48 \\
\hline$\alpha 5$ & 0.36 & 0.82 & 0.44 & & & $0.6^{d}$ & 0.05 & 0.73 & 29 \\
\hline$\alpha 6^{e}$ & 0.41 & 1.0 & 0.41 & & & & & & \\
\hline$\alpha 7$ & 0.34 & 0.85 & 0.40 & & & $\leq 0.6^{b}$ & 0.07 & 0.71 & $\geq 15$ \\
\hline
\end{tabular}

${ }^{a}$ Solutions degassed with nitrogen or argon by bubbling. $k$ 's are $10^{9} \mathrm{~s}^{-1}$ and $\alpha 7$ is the di- $n$-butyl-substituted $\alpha 7$. The lifetime of thiophene $(\alpha 1)$ is $\sim 1 \mu \mathrm{s}$ (by sensitization from xanthone). ${ }^{b}$ Assumes value in benzene. ${ }^{c}$ Assumes $\left(1-\phi_{\mathrm{T}}-\phi_{\mathrm{F}}\right)=0.005 .{ }^{d}$ Assumes average of values in acetonitrile and benzene. ${ }^{e} \alpha 6$ is "not soluble" in methylcyclohexane, acetonitrile, and ethanol.

TABLE 3: Absorption, Fluorescence, Fluorescence Rate Constants and Triplet-Triplet Data in Dioxane ${ }^{a}$ at Room Temperature

\begin{tabular}{|c|c|c|c|c|c|c|}
\hline cpd & abs $\max \lambda_{\max } / \mathrm{nm}$ & $\epsilon / \mathrm{mol}^{-1} \mathrm{dm}^{3} \mathrm{~cm}^{-1}$ & $k_{\mathrm{F}}^{0}(\mathrm{SB}) / 10^{9} \mathrm{~s}^{-1}$ & $k_{\mathrm{F}}^{0} / 10^{9} \mathrm{~s}^{-1}$ & fluoresc $\max ^{b}$ & $\mathrm{~T}-\mathrm{T} \max ^{a}$ \\
\hline$\alpha 1$ & 231 & $8340^{c}$ & $\begin{array}{l}0.33^{d} \\
0.023^{d}\end{array}$ & & & 305 \\
\hline$\alpha 2$ & 303 & 12440 & & $\begin{array}{l}0.22^{e} \\
0.38^{e}\end{array}$ & 362 & 385 \\
\hline$\alpha 3$ & 354 & $22080^{f}$ & 0.30 & 0.31 & 407,426 & 460 \\
\hline$\alpha 4$ & 392 & $31560^{g}$ & 0.34 & 0.36 & 437,478 & 560,700 \\
\hline$\alpha 5$ & 417 & 42670 & 0.38 & 0.44 & 482,514 & 630 \\
\hline$\alpha 6$ & 436 & 47910 & & 0.41 & 502,537 & 685 \\
\hline$\alpha 7^{h}$ & 441 & 50500 & 0.39 & 0.40 & 522,560 & 720 \\
\hline
\end{tabular}

${ }^{a} \mathrm{~T}-\mathrm{T}$ maxima in other solvents vary by only $10 \mathrm{~nm}$ at the maximum. The $\alpha 1$ datum is with acetonitrile. ${ }^{b}$ The italicized wavelength is the band maximum. The other one is another distinct band (see Figure 4). The $\alpha 1$ does not fluoresce but shows phosphorescence (see text). ${ }^{c}$ Assumes same value as for ethanol. ${ }^{d}$ First value using Försters equation instead of Strickler-Berg (SB) with integration from $38000 \mathrm{~cm}^{-1}$ (263 nm) to 48000 $\mathrm{cm}^{-1}(208 \mathrm{~nm})$. Second (lower) value from integration over entire first absorption band (same wavelength/wavenumber) limits but different $\nu_{0}$ (absorption maximum value). ${ }^{e}$ First (upper) value using a $\tau_{\mathrm{F}}$ from cyclohexane $(0.081 \mathrm{~ns})$ and second one using $\tau_{\mathrm{F}}$ value in benzene of $0.046 \mathrm{~ns}$ with a different instrument. First value has more potential error because normal $\tau$ lower limit is $\geq 100$ ps with the instrument used. ${ }^{f} \epsilon($ benzene $)=$ $22540 \mathrm{M}^{-1} \mathrm{~cm}^{-1}, \epsilon$ (acetonitrile) $=24215 \mathrm{M}^{-1} \mathrm{~cm}^{-1}$ and $\epsilon($ ethanol $)=23590 \mathrm{M}^{-1} \mathrm{~cm}^{-1}$ at their maxima of 354,352 , and $355 \mathrm{~nm}$, respectively. ${ }^{g} \epsilon$ (acetonitrile) is 34700 at its maximum. ${ }^{h}$ For the di- $n$-butyl-substituted $\alpha 7$.

as well-for example, $\phi_{\mathrm{F}}$ and $\phi_{\mathrm{T}}$ of $\alpha 3-\alpha 5$ vs $n$ if used to predict $\alpha 2, \alpha 6$, and $\alpha 7$.

The room temperature solution fluorescence of $\alpha 2-\alpha 7$ is shown in Figure 5. Note that there is definitely more resolution than for absorption. Table 3 gives representative fluorescence spectra maxima in dioxane. As in the case of absorption, there is little solvent effect on fluorescence. Note in Figure 5 at 77 $\mathrm{K}$ (as well as in ref 1 ) that there is some considerable increase in vibrational resolution compared to that at room temperature, particularly noticeable for $\alpha 2$, less for $\alpha 3$, and continuing to be less as the number of rings increases to $\alpha 4$ and on to $\alpha 7$. Of notable importance is the fact that there is almost no red shift $\left(100-200 \mathrm{~cm}^{-1}\right)$ in the fluorescence maxima between room temperature and $77 \mathrm{~K}$. This is in marked contrast to that of absorption (see above). Note also that there can be a switch between the first two bands regarding which one is the maximum at room temperature as the number of rings increases (from the second band to first band from $\alpha 4$ on) and between room- and low-temperature $(77 \mathrm{~K})$ bands for a given $\alpha n$-for example, see $\alpha 4$ in particular. We have also found an excellent correlation between the energy of the first (and second) band of fluorescence vs $1 / n$ (or $1 / N$ ).

We were unable to see phosphorescence in three different laboratories for $n=2$ or $n=3$ at $77 \mathrm{~K}$ in various glasses 


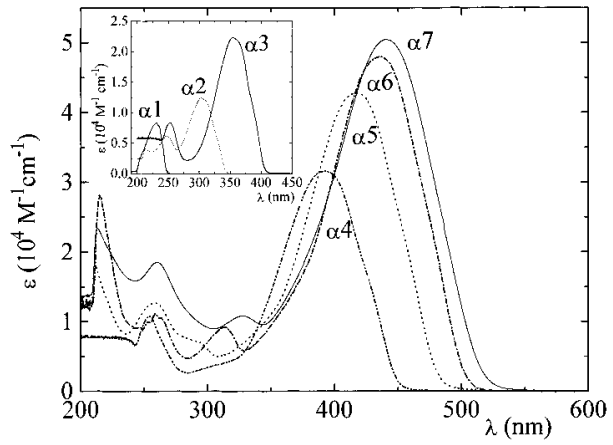

Figure 1. Room temperature absorption spectra of $\alpha 1-\alpha 7$ in dioxane.
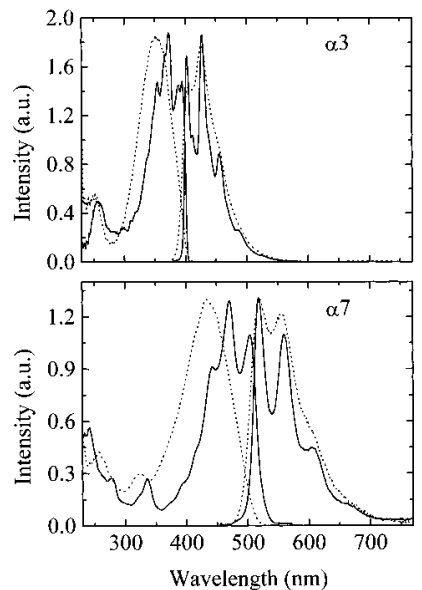

Figure 2. Absorption and fluorescence at room temperature $(\cdots)$ and fluorescence and fluorescence excitation spectra (-) at $77 \mathrm{~K}$ for $\alpha 3$ and $\alpha 7$ in ethanol.

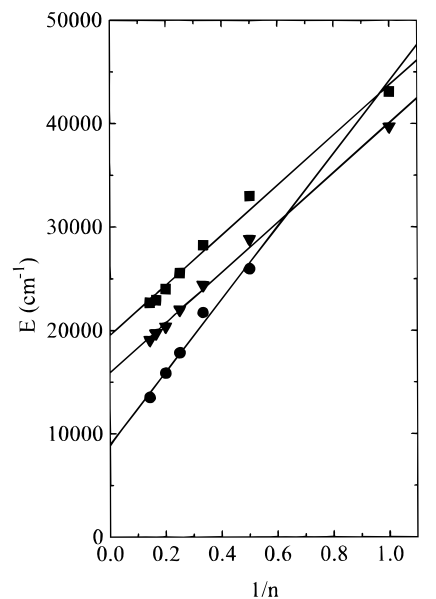

Figure 3. $E_{\mathrm{T}_{1}-\mathrm{T}_{\mathrm{n}}}(\max ) \bullet, E_{\mathrm{S}_{0}-\mathrm{S}_{1}}(\max ) \boldsymbol{\square}$, and $E_{\mathrm{S}_{0}-\mathrm{S}_{1}}(0-0) \boldsymbol{\nabla}$ vs $1 / n$.

including an aliphatic hydrocarbon, ethanol, and one containing $10 \%$ ethyl iodide, and others could not see phosphoresecence for $\alpha 3$ and $\alpha 4 .^{1,26-28}$ Particularly, $n=2$ (as well as $n=3$ ) emission(s) should be quite easy to observe even with a quantum yield of $10^{-3}$ (or even lower), since they are expected to emit in a spectral region $(500-700 \mathrm{~nm}$ ) easily detectable by sensitive photomultiplier tubes. Also, no phosphorescence was observed for dodecyl-substituted $\alpha 6, \alpha 7$, and longer ones. ${ }^{27}$ However, it has been reported ${ }^{29}$ that $\alpha 3$ (in undefined environmental and temperature conditions) showed a sharp, single band phosphorescence at $826 \mathrm{~nm}$, which was stated to be identical with that in shape and energy of poly(3-hexylthiophene) in a thin film at $18 \mathrm{~K}$. This phosphorescence emission from $\alpha 3$ seems very strange particularly from the point of view that it is at the same wavelength as a multithiophene component polymer-surely the

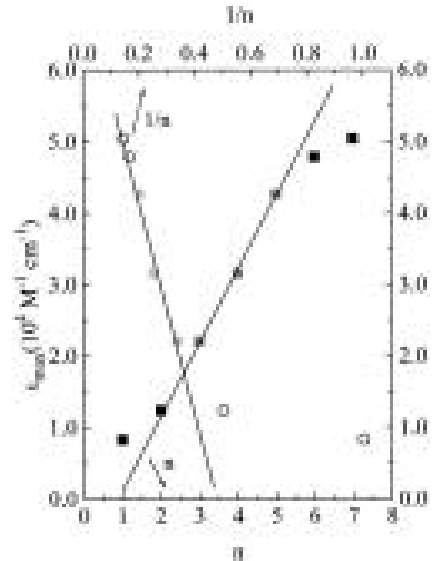

Figure 4. Extinction coefficients $(\epsilon)$ in dioxane vs $1 / n(\bigcirc)$ and $n(\square)$.
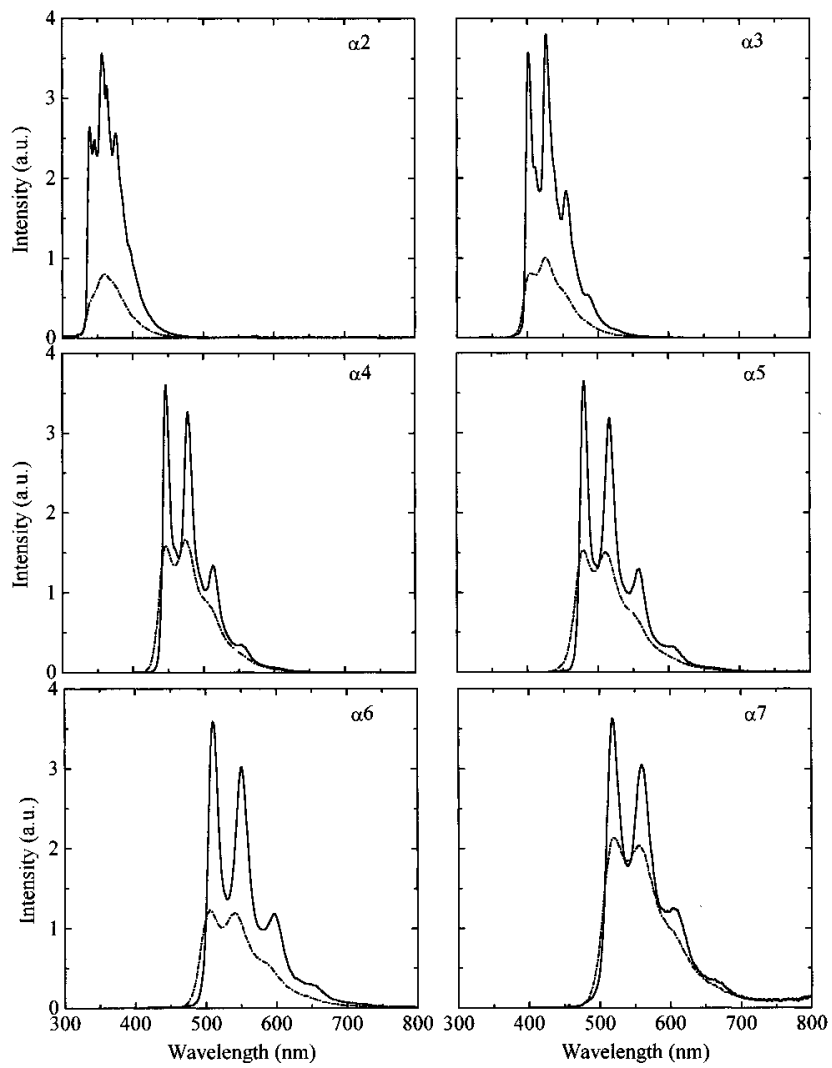

Figure 5. Room- (- - ) and low-temperature (77 K) (-) fluorescence spectra of $\alpha 2-\alpha 7$ in ethanol, except for $\alpha 6$ where the solvent is 2-methyltetrahydrofuran.

triplet energies could not be the same. Furthermore, there is some published evidence that the lowest triplet of $\alpha 3$ is near $720 \mathrm{~nm}(0-0)^{30}$ and we have evidence that it may be at still lower in energy at $\sim 788 \mathrm{~nm}(0-0)$ based on photoacoustical experiments. Presumably, $\alpha 3$ could possibly have a maximum at such a wavelength $(826 \mathrm{~nm})$, but then surely the polymer must be at considerably longer wavelength.

Very interestingly, we did see a weak phosphorescence of thiophene, $\alpha 1$, with a maximum at $\sim 430 \mathrm{~nm}$ and a $0-0$ band near $362 \mathrm{~nm}\left(27600 \mathrm{~cm}^{-1}\right)$. Others ${ }^{31,32}$ found the lowest $\mathrm{S}_{0} \rightarrow$ $\mathrm{T}_{1}$ absorption by electron impact to have an onset near 370$375 \mathrm{~nm}$. Thus, there is excellent agreement for the energy origin $(0-0)$ of the lowest triplet state being very close to $27300 \mathrm{~cm}^{-1}$. This would give a singlet - triplet $\left(\mathrm{S}_{1}-\mathrm{T}_{1}\right)$ separation of close to $12300 \mathrm{~cm}^{-1}$. For $\alpha 3$ using $720 \mathrm{~nm}^{30}\left(\sim 13900 \mathrm{~cm}^{-1}\right)$ as the origin of $T_{1}$, there is a $S_{1}-T_{1}$ separation of $10800 \mathrm{~cm}^{-1}$, 


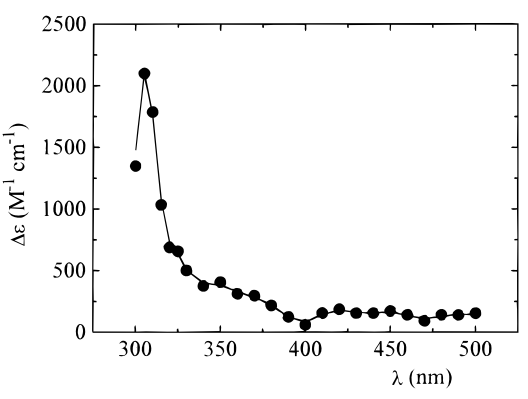

Figure 6. Triplet-triplet absorption spectrum of $\alpha 1$ at room temperature in benzene.

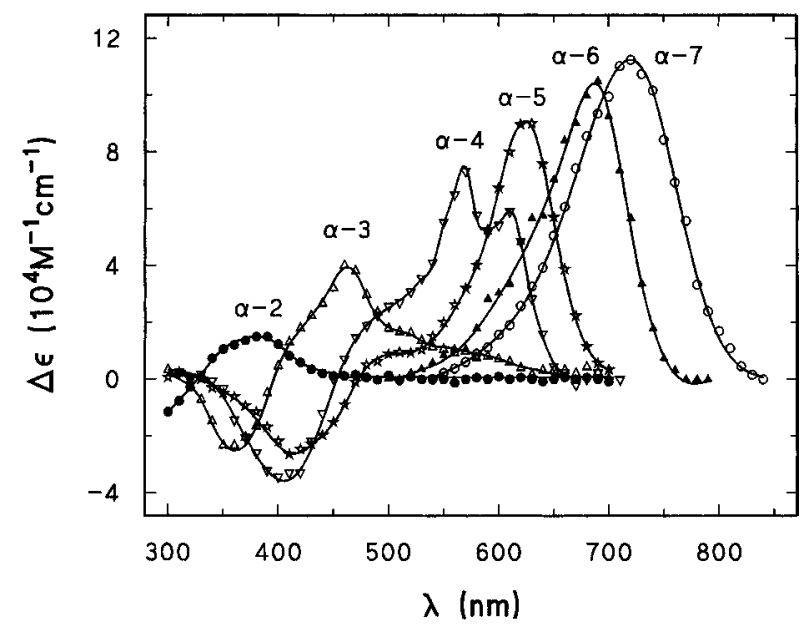

Figure 7. Triplet-triplet absorption spectra of $\alpha 2-\alpha 7$ at room temperature in benzene.

whereas using $788 \mathrm{~nm}\left(\sim 12700 \mathrm{~cm}^{-1}\right)$, the separation is $12000 \mathrm{~cm}^{-1}$, close to that of thiophene.

Triplet-Triplet Absorption. Our triplet-triplet absorption data are shown in Figure 6 for $\alpha 1$ and in Figure 7 for $\alpha 2-\alpha 7$. Table 3 gives representative spectral data in dioxane. As before, there is little effect of solvent on these values. Note again the shift of the absorption maximum to progressively longer wavelengths as the number of rings increase. We also consider this point in Figure 3 where the energy of the $\mathrm{T}-\mathrm{T}$ maxima are plotted as a function of $1 / n$. Thiophene has a weak shoulder at a wavelength longer than that of the maximum, near 360 $\mathrm{nm}$, and $\alpha 3$ and $\alpha 4$ clearly have band shoulders (bands for $\alpha 4$ ) at wavelengths both shorter and longer than that of the main maximum. Also, the onset of the $460 \mathrm{~nm}$ band maximum of $\alpha 3$ is at an unusually long wavelength, essentially equal to that of $\alpha 5$ (where the maximum is at $630 \mathrm{~nm}$ ). It appears that for $\alpha 3$ there must be more than one $\mathrm{T} \rightarrow \mathrm{T}_{n}$ transition in the broad absorption region of $\sim 700-460 \mathrm{~nm}$, and probably for $\alpha 4$ also. The extinction coefficient of the principal maximum progressively increases as $n$ increases. Some other data exist for some of the individual oligomers. ${ }^{1,4,26-28,30}$ For different alkylsubstituted $\alpha 9$ and $\alpha 11, \mathrm{~T}_{1}-\mathrm{T}_{n}$ maxima occur at 774 and 805 nm, respectively. ${ }^{27}$ The longer (dodecyl) alkyl chain substitutions cause a blue shift in the maxima compared to shorter ones (butyl).

Quantum Yields, Lifetimes, and Rate Constants. The $\phi_{\mathrm{F}}$ and $\phi_{\mathrm{T}}$ values are remarkably solvent independent (Tables 1 and 2), but $\phi_{\mathrm{F}}$ clearly increases and $\phi_{\mathrm{T}}$ clearly decreases as the number of rings increase and both become essentially constant from $\alpha 5$ to $\alpha 7$ (also see Figure 8 ). The $\tau_{\mathrm{F}}$ values are also quite solvent independent, but clearly, the $\tau_{\mathrm{F}}$ increases as the number of rings increase, becoming essentially constant from $\alpha 5$ to $\alpha 7$. The $k_{\mathrm{F}}^{0}=1 / \tau_{\mathrm{F}}^{0}$ values, where $\tau_{\mathrm{F}}^{0}=\tau_{\mathrm{F}} / \phi_{\mathrm{F}}$, derived from $\phi_{\mathrm{F}}$ and $\tau_{\mathrm{F}}$ data in Tables 1 and 2 are quite constant as a function of the

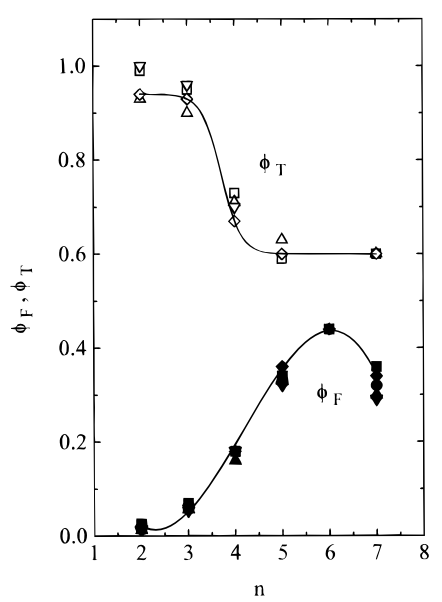

Figure 8. $\phi_{\mathrm{F}}$ (dark symbols) and $\phi_{\mathrm{T}}$ (open symbols) vs $n$ for $\alpha 2-\alpha 7$ in four solvents.

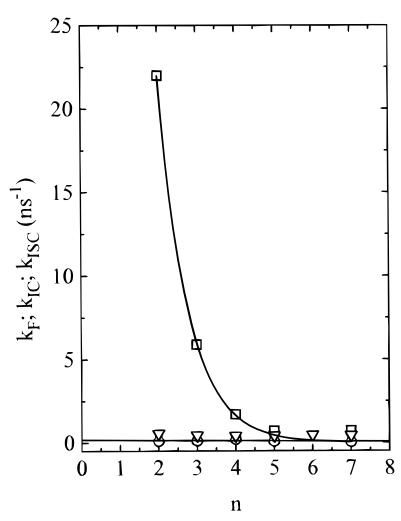

Figure 9. $k_{\mathrm{F}}(\nabla), k_{\mathrm{ISC}}(\square)$, and $k_{\mathrm{IC}}(\bigcirc)$ vs $1 / n$ for $\alpha 2-\alpha 7$ in benzene.

number of rings except perhaps for $\alpha 2$. Also, for $\alpha 3, k_{\mathrm{F}}^{0}$ (in Table 2) seems somewhat different (lower) than for $\alpha 4-\alpha 7$, except for perhaps benzene and acetonitrile. The $k_{\mathrm{F}}^{0}$ values in benzene generally appear larger than in the other solvents (Tables 1 and 2). Despite this, it is generally valid that $k_{\mathrm{F}}^{0}$ is quite constant as a function of both solvent and the number of rings. The $k_{\mathrm{NR}}$ values show a clear decreasing trend with increasing number of rings (Tables 1 and 2), but apparently, $k_{\mathrm{NR}}$ attains a nearly constant value from $\alpha .5$ to $\alpha 7$. Since we have all the photophysical data required, we can further determine both $k_{\mathrm{ISC}}=\phi_{\mathrm{T}} / \tau_{\mathrm{F}}$ and $k_{\mathrm{IC}}=\left(1-\phi_{\mathrm{F}}-\phi_{\mathrm{T}}\right) / \tau_{\mathrm{F}}$, which are shown in Tables 1 and 2. It is clear that $k_{I S C}$ decreases with increasing number of rings, reaching a constant value from $\alpha 5$ to $\alpha 7$. The trend in $k_{\mathrm{IC}}$ is much more difficult to discern, but from $\alpha 2$ to $\alpha 5-\alpha 7$, there is a decrease in $k_{\mathrm{IC}}$ with the data for acetonitrile, while the other solvents produce a scatter on the way from $\alpha 2$ to $\alpha 5-\alpha 7$. All the above rate constants are illustrated in Figure 9. The reason for the scatter of $k_{\mathrm{IC}}$ is that $k_{\mathrm{IC}}$ depends on the difference $1-\phi_{\mathrm{T}}-\phi_{\mathrm{F}}$ and $\phi_{\mathrm{T}}+\phi_{\mathrm{F}}$ is close to 1 for $\alpha 2$ and $\alpha 3$. Moreover, the error in $\phi_{\mathrm{T}}$ is $\pm 15 \%$ so that the $k_{\mathrm{IC}}$ value can be very sensitive to this (where $\phi_{\mathrm{T}}=$ $0.90-0.99$ and a $2 \%$ change in $\phi_{\mathrm{T}}$ results in a minimum of a $300 \%$ change in $\left.k_{\mathrm{IC}}\right)$. This is not true for $k_{\mathrm{ISC}}$ where the change expected in the $k_{\mathrm{ISC}}$ is essentially no more than about the same as the error in $\phi_{\mathrm{T}}\left(\phi_{\mathrm{T}}= \pm 15 \%\right.$, only $\sim 7 \%$ error in $\left.\tau_{\mathrm{F}}\right)$.

Our lifetimes of fluorescence data are given in Tables 1 and 2 , and other fluorescence lifetime data also exist for some of the oligomers. Data on $\alpha 3-\alpha 6^{2-4}$ are within a few percent to $10 \%$ of ours. $^{2}$ Other data ${ }^{33}$ for $\alpha 3$ and $\alpha 6$ are $\sim 30 \%$ shorter than ours (theirs in dichloromethane), and for others ${ }^{34}$ the $\alpha 3$ is some $30 \%$ longer than ours while $\alpha 4$ is close to ours. The 
$\alpha 5$ in rigid poly(methyl methacrylate) has $\tau_{\mathrm{F}}=0.9 \mathrm{~ns},{ }^{4}$ similar to ours in five solvents.

Recently, others have measured some photophysical parameters but there is no complete set even on any one compound; commonly, data on $\alpha 2$ are missing, and there is no data on $\alpha 7$. Our $\phi_{\mathrm{F}}$ and $\tau_{\mathrm{F}}$ data are given in Tables 1 and 2 . The $\phi_{\mathrm{F}}$ values of $\alpha 3-\alpha 6$ have been determined by Chosrovian et al. ${ }^{2}$ and Colditz et al. $^{3}(\alpha 2-\alpha 6)$, and generally they are within $10 \%$ of our values. The former authors believed that $k_{\mathrm{NR}}$ was largely due to $S_{1} \rightarrow S_{0}$ internal conversion. However, this was based on $\phi_{\mathrm{T}}$ values of approximately 0.2 for $\alpha 3$ and $\alpha 4$, which are certainly not correct (see later discussion). Others ${ }^{3,4}$ have measured $\phi_{\mathrm{F}}$ of $\alpha 2-\alpha 6$, and except for $\alpha 2$, these were also within $10 \%$ of our values. Moreover, $\phi_{\mathrm{F}}$ data $^{33}$ are available on $\alpha 2-\alpha 4$ and $\alpha 6$ and all agree with ours except for $\alpha 6$ (which is lower than ours and that of others given above). In addition, some data are available on the scattering of compounds: $\phi_{\mathrm{F}}$ of $\alpha 3$ is similar to ours and others ${ }^{26,30,34}$ but $\alpha 4$ is noticeably lower ${ }^{34}$ than ours and that of others ${ }^{2-4,26}$ (and $\tau_{\mathrm{F}}$ 's are reasonably similar to ours).

Triplet yields for $\alpha 3$ of $0.2,{ }^{26} \geq 0.9,{ }^{30}$ and $0.95^{35}$ exist vs our values of $0.90-0.96$ (Tables 1 and 2). Based on all available data, a $\phi_{\mathrm{T}}$ for $\alpha 3$ of 0.2 cannot be correct. For $\alpha 4$, a value of $\sim 0.2^{26}$ exists for $\phi_{\mathrm{T}}$ vs our values of $0.63-0.73$ (Tables 1 and 2). On the basis of our $\phi_{\mathrm{T}}$ data and $\phi_{\Delta}$ for $\alpha 4(0.69),{ }^{4}$ we believe the value of $\sim 0.2$ for $\phi_{\mathrm{T}}$ of $\alpha 4$ is not correct.

In Tables 1 and 2 our triplet state lifetimes are shown. Some other data also exist: for $\alpha 3$, a value of $30 \mu \mathrm{s}$ in methanol at zero laser dose and concentration has been given. ${ }^{28}$ Nonetheless, this is much shorter than our values ranging from $\sim 62$ (acetonitrile) to $108 \mu$ s (dioxane) (Table 2). For $\alpha 3$ and $\alpha 4$, others $^{26}$ report a value of $57(\alpha 3)$ and $45 \mu$ s $(\alpha 4)$ in ethanol vs our values of $91(\alpha 3)$ and $43 \mu \mathrm{s}(\alpha 4)$ in the same solvent. The lifetime of $\alpha 5$ in dioxane in nitrogen has been given ${ }^{4}$ to be 7.7 $\mu \mathrm{s}(0.24 \mu \mathrm{s}$ in air), which is considerably shorter than our value in dioxane and all other solvents (Tables 1 and 2). Based on induced dichroism decay, the decay of the triplet of $\alpha 3$ in a viscous medium has been given to be a few nanoseconds. ${ }^{36}$ Presumably, oxygen was present, but nonetheless, this is an unusually short lifetime for a highly viscous medium even in the presence of oxygen. Lifetimes of 1- to 3-dodecyl-substituted $\alpha 6, \alpha 7, \alpha 9$, and $\alpha 11$ were determined in frozen 2-methyltetrahydrofuran at $80 \mathrm{~K}$ by monitoring the photoinduced absorption assigned as $\mathrm{T}_{1}-\mathrm{T}_{2}$ absorption. ${ }^{27}$ Lifetimes were $380-470 \mu \mathrm{s}$ for $\alpha 6,350-380 \mu$ s for $\alpha 7,300 \mu$ s for $\alpha 9$, and $250 \mu$ s for $\alpha 11$. The trend to shorter lifetimes with increasing number of rings is the same as what we find for $\alpha 2-\alpha 7$ (Tables 1 and 2). An interesting case is that of $\alpha 2$ in a seeded free-jet expansion where the triplet lifetime has been given as $550 \mathrm{~ns}{ }^{37}$ This is extremely short compared to our values of $100-146 \mu \mathrm{s}$ ( $\sim 225$-fold) at room temperature in fluid solution. This also seems to be very short for a triplet of an isolated, cold molecule (of $\alpha 2$ ) (see later discussion).

Calculations, Geometry, Stability of Conformers, and Transition Energies. We have done an extensive number (10) of different types of calculations and at a number of levels including three at the $a b$ initio level regarding the relative stability of the all-cis and all-trans conformers of $\alpha 2$ (Table 4), as well as $\alpha 3-\alpha 5$ (Table 5). In Table 4, for $\alpha 2$ we compared a number of calculations of the literature with those of ours and proposed $\Delta H$ values between cis and trans. We did significantly more and higher level calculations on $\alpha 3$ and $\alpha 5$, since their was very little or no significant data on these latter $\alpha$-oligothiophenes. It can be seen in Table 4 that for $\alpha 2$, the two experimental $\Delta H$ values are quite different from one
TABLE 4: Relative Stabilities of Cis and Trans Conformers of $\alpha 2$ by Various Theoretical Approximations ${ }^{a}$

\begin{tabular}{lll}
\hline \multicolumn{1}{c}{ method } & \multicolumn{1}{c}{ result $(\mathrm{kcal} / \mathrm{mol})$} & ref/footnote \\
\hline exptl & $\mathrm{t}<\mathrm{c}, 1.16 \pm 0.13^{b} ; 0.2$ & $41,32,39,40$ \\
MM2 & $\mathrm{t}<\mathrm{c}, 1.04$ & this work \\
MOPAC & $\mathrm{c}<\mathrm{t}, 0.46$ & this work \\
AMPAC/AM1 & $\mathrm{t}<\mathrm{c}, 0.16$ & this work \\
AMPAC/AM1 & $\mathrm{t}<\mathrm{c}, 0.25$ & 54 \\
ab initio STO 3G & $\mathrm{t}<\mathrm{c}, 1.3$ & $c$ \\
ab initio STO 3G & $\mathrm{t}<\mathrm{c}, 1.20$ & $d$ \\
ab initio 3-21G* & $\mathrm{t}<\mathrm{c}, 0.64$ & $e$ \\
ab initio RHF/DPZ & $\mathrm{t}<\mathrm{c}, 0.8$ & $f$ \\
plus electron correlation & $\mathrm{t}<\mathrm{c}, 0.4$ & $f$ \\
ab initio 3-21G & $\mathrm{t}<\mathrm{c}, 1.9$ & $f$ \\
ab initio 3-21G, 631 & $\mathrm{t}<\mathrm{c}, 0.6-0.7$ & 40 \\
Columbus ACPF gradient & $\mathrm{t}<\mathrm{c}, 0.5$ & $g$
\end{tabular}

${ }^{a}$ See Table 5 for more details on our calculations regarding other $\alpha$-oligothiophenes and their conformers. ${ }^{b}$ See text for a possible caveat regarding whether this truly represents the $\Delta H$ of cis and trans conformers (ref 41). ${ }^{c}$ Bredas, J. L.; Street, G. B.; Thiemans, B.; Andre, J. M. J. Chem. Phys. 1985, 83, 1323. ${ }^{d}$ Jones, D.; Guerra, M.; Favaretto, L.; Modelli, A.; Fabrizio, M.; Distefano, G. J. Phys. Chem. 1990, 94, 5761. ${ }^{e}$ Distefano, G.; Colle, D.; Jones, D.; Zambianchi, M.; Favaretto, L.; Modelli, A. J. Phys. Chem. 1993, 97, 3504. ${ }^{f}$ Quattrocchi, C.; Lazzaroni, R.; Bredas, J. L. Chem. Phys. Lett. 1993, 208, 120. ${ }^{g}$ Kofranck, M.; Kovar, T.; Lischka, H.; Karpfen, A. J. Mol. Struct. 1992, 259, 181.

TABLE 5: Relative Stability of the Conformers All-Cis and All-Trans $\alpha$-Oligothiophenes (this Work)

\begin{tabular}{|c|c|c|c|}
\hline cpd & method $^{a, b}$ & rel stability & $\Delta H$ cis $-\operatorname{trans}(\mathrm{kcal})$ \\
\hline \multirow[t]{4}{*}{$\alpha 2$} & MOPAC & $\mathrm{c}<\mathrm{t}$ & 0.46 \\
\hline & MM2 & $\mathrm{t}<\mathrm{c}$ & 1.04 \\
\hline & AMPAC/AM1 & $\mathrm{t}<\mathrm{c}$ & 0.16 \\
\hline & exptl & $\mathrm{t}<\mathrm{c}$ & $1.16 \pm 0.13 ; ;^{c} 0.2$ \\
\hline \multirow[t]{10}{*}{$\alpha 3$} & MOPAC & $\mathrm{cc}<\mathrm{tt}$ & 0.89 \\
\hline & AMPAC/AM1 & $\mathrm{tt}<\mathrm{cc}$ & 0.35 \\
\hline & MM2 & $\mathrm{tt}<\mathrm{cc}$ & 2.12 \\
\hline & MNDO 93, AM1 & $\mathrm{tt}<\mathrm{cc}$ & 0.42 \\
\hline & MNDO 93, PM3 & $\mathrm{cc}<\mathrm{tt}$ & 0.83 \\
\hline & DGauss-LDF & $\mathrm{tt}<\mathrm{cc}$ & 1.39 \\
\hline & DGauss-NLSD & $\mathrm{tt}<\mathrm{cc}$ & 1.18 \\
\hline & ab initio STO $3 \mathrm{G}$ & $\mathrm{tt}<\mathrm{cc}$ & 2.41 \\
\hline & ab initio $6-311 \mathrm{G}$ & $\mathrm{tt}<\mathrm{cc}$ & 2.22 \\
\hline & $a b$ initio $6-31 \mathrm{G}(\mathrm{d})$ & $\mathrm{tt}<\mathrm{cc}$ & 1.47 \\
\hline$\alpha 4$ & AMPAC/AM1 & $\mathrm{ttt}<\mathrm{ccc}$ & 2.73 \\
\hline \multirow[t]{8}{*}{$\alpha 5$} & MOPAC & $\operatorname{cccc}<\mathrm{tttt}$ & 1.54 \\
\hline & AMPAC/AM1 & $\mathrm{tttt}<\operatorname{cccc}$ & 1.41 \\
\hline & MM2 & $\mathrm{tttt}<\mathrm{cccc}$ & 8.4 \\
\hline & MNDO 93, AM1 & $\mathrm{tttt}<\mathrm{cccc}$ & 1.72 \\
\hline & MNDO 93, PM3 & $\operatorname{cccc}<\mathrm{tttt}$ & 1.52 \\
\hline & DGauss $\cdot$ LDF & $\mathrm{tttt}<\mathrm{cccc}$ & 6.46 \\
\hline & DGauss $\cdot N L S D$ & $\mathrm{tttt}<\operatorname{cccc}$ & 5.79 \\
\hline & $a b$ initio STO $3 \mathrm{G}$ & $\mathrm{tttt}<\operatorname{cccc}$ & 4.81 \\
\hline
\end{tabular}

${ }^{a}$ Gaussian 92 code was used for all ab initio calculations. For one of them, 6-31G(d), a polarization function (d-orbital) was added for C and $\mathrm{S}$ atoms. For DGauss, double- $\zeta$ potential basis sets were used for orbital functions and a nonlocal spin density (NLSD) correction was used in one of the calculations with DGauss. ${ }^{b}$ For MNDO 93 (AM1 or PM3) and $a b$ initio STO 3G calculations, initial input geometries were from MM2. For DGauss, input geometries were planar. For $a b$ initio 6-311 G and 6-31G(d), input geometries were from STO3G optimized geometries (planar). A second set of calculations using MNDO 93, AM1 geometry as input gave $\sim 0.2$ kcal difference from planar input but did not change the relative stability. ${ }^{c}$ See text for possible caveat regarding whether this truly represents the $\Delta H$ of cis and trans conformers.

another. This potentially complicates the comparison with theory. Nonetheless, it appears that the trans conformer of $\alpha 2$, albeit twisted around the inter-ring bond, is lower in energy. ${ }^{32,38-40}$ Note that MOPAC and MNDO93,PM3 incorrectly predict the 
cis conformer as the more stable one. Also, the presumably less sophisticated MM2 approach predicts $\Delta H$ in the same vicinity as several of the other methods including the $a b$ initio level calculations.

Some literature data propose that for $\alpha 2$ in the ground state, both cis and trans conformers coexist in an $\alpha 2$-seeded free-jet expansion and that the inter-ring twisting is about the same in both conformers. ${ }^{41}$ The $\Delta H$ between cis and trans was given to be $1.16 \pm 0.13 \mathrm{kcal} / \mathrm{mol}\left(406 \pm 46 \mathrm{~cm}^{-1}\right)$ with the trans conformer being the more stable. On the other hand, different but parallel supersonic jet experiments ${ }^{42}$ involving $\alpha 2$ and using the same emission techniques, plus hole burning, point to the existence of a torsionally twisted equivalent pair (a double minimum) around the trans conformer in the ground state in place of identifying the coexistence of cis and trans conformers as done earlier. ${ }^{41}$ The equilibrium structure (trans) existed with a twist of $\sim 21^{\circ}$ from planarity and with a barrier of $\sim 25 \mathrm{~cm}^{-1}$ between the two minima (at $21^{\circ}$ ). In the first excited state by contrast, $\alpha 2$ was found to be trans and planar with a deep, steep single minimum torsional potential well around the equilibrium structure. As we will see later, our spectroscopic data strongly indicate a similar situation in the first excited state. The difference in the supersonic jet experiments ${ }^{41,42}$ makes it difficult to be certain that the proposed ${ }^{41} \Delta H(1.16 \mathrm{kcal})$ between ground state cis and trans is actually appropriate.

For $\alpha 3$, the higher level calculations (DGauss and several levels of $a b$ initio) predict the tt conformer as the more stable as does the MM2 approach and with a fairly comparable $\Delta H$ (Table 5). If all three conformers are calculated (tt, ct, cc), then $\mathrm{tt}$ is still the most stable except by MOPAC and MNDO93,PM3. The $\Delta H$ between $t$ and cc in general seems to be in the $2.0 \mathrm{kcal} / \mathrm{mol}$ range $( \pm 0.4)$ for ab initio methods and $\sim 1.2 \mathrm{kcal} /$ mol for the DGauss approach (lower for AMPAC and MNDO, AM1) (Table 5). Again, MOPAC and MNDO93,PM3 very likely predict stability in the wrong order, $\mathrm{cc}<\mathrm{tt}$.

For $\alpha 4$ and $\alpha 5$, all methods except MOPAC and MNDO93,AM1 predict the all-t conformer as the most stable. The $\Delta H$ beween the all-trans and all-cis conformer varies according to the calculation method (Table 5). If we consider all possible conformers of $\alpha 5$ (10 of them), the all-trans conformer is still the most stable where generally the greater the number of adjacent cis pairs, the higher the relative energy.

We have determined the dihedral angle of all the conformers of $\alpha 3$ and for the all-cis and all-trans conformers for $\alpha 5$. All ab initio STO3G, DGauss (with planar input), and MM2 predict either planar ( $a b$ initio STO3G) or near planar geometry (essentially no twisting) for the cc and tt conformers of $\alpha 3$. This is also true for MOPAC, but remember, it predicts the cc conformer as the more stable vs tt for the other calculations considered here (and all the rest except MNDO93,PM3). X-ray data of trithiophene $(\alpha 3)^{43}$ give a clear indication that the $t$ is the more stable, that is, this is the conformeric form in the crystal with inter-ring angles of about $6^{\circ}-9^{\circ}$. Also, a dibutylsubstituted trithiophene has a tt configuration ${ }^{44}$ with a higher angle of inter-ring twist than for $\alpha 3$ itself (not unexpected). However, the barrier to rotation was given to be $19.7 \mathrm{kcal} / \mathrm{mol}$ in the ground state and $4.2 \mathrm{kcal} / \mathrm{mol}$ in the lowest excited singlet state using ${ }^{1} \mathrm{H}$ NMR. This seemed quite incompatible with a "single" inter-ring bond in the ground state and a more double inter-ring bond in the lowest excited singlet state. Very recently, reinvestigation ${ }^{44}$ of the same molecule at higher resolution now suggests a ground state barrier of $0.5-1 \mathrm{kcal} / \mathrm{mol}$ with the excited state barrier similar to that reported earlier. ${ }^{44}$ These new data are now compatible with our findings (see later discussion). In the case of $\alpha 5$, only STO3G gives planar
TABLE 6: Calculated and Experimental First Transition Energies of Some $\alpha$-Oligothiophenes ${ }^{a}$

\begin{tabular}{|c|c|c|c|c|}
\hline \multirow[b]{2}{*}{ cpd } & \multirow{2}{*}{$\begin{array}{l}\text { geometry } \\
\text { opt method }\end{array}$} & \multirow[b]{2}{*}{ conformer } & \multicolumn{2}{|c|}{ transition energy, ${ }^{b} \mathrm{~nm}$} \\
\hline & & & theory $(f)$ & $\operatorname{exptl}^{c}$ \\
\hline \multirow[t]{6}{*}{ di- } & $\operatorname{MM} 2\left(\mathrm{~S}, \mathrm{sp}^{2}\right)$ & $\mathrm{t}$ & $348(0.70)$ & $302(303)$ \\
\hline & & $\mathrm{c}$ & $345(0.72)$ & \\
\hline & MOPAC & $\mathrm{t}$ & $336(0.65)$ & $302(303)$ \\
\hline & & c & $335(0.66)$ & \\
\hline & AMPAC/AM1 & $\mathrm{t}$ & 345 & $302(303)$ \\
\hline & & $\mathrm{c}$ & 325 & \\
\hline \multirow[t]{4}{*}{ tri- } & MOPAC & all-t & $398(1.07)$ & $350(354)$ \\
\hline & & all-c & 397 (1.09) & \\
\hline & AMPAC/AM1 & all-t & 373 & $350(354)$ \\
\hline & & all-c & 354 & \\
\hline \multirow[t]{2}{*}{ tetra- } & AMPAC/AM1 & all-t & 398 & $386(392)$ \\
\hline & & all-c & 333 & \\
\hline \multirow[t]{7}{*}{ quinque- $^{d}$} & $\operatorname{MM} 2\left(\mathrm{~S}, \mathrm{sp}^{2}\right)$ & all-t & $431(1.65)$ & $410(417)$ \\
\hline & & all-c & $480(1.68)$ & \\
\hline & MOPAC & all-t & $422(1.59)$ & $410(417)$ \\
\hline & & all-c & $473(1.60)$ & \\
\hline & AMPAC/AM1 & all-t & 405 & $410(417)$ \\
\hline & & all-c & 355 & \\
\hline & STO $3 \mathrm{G}$ & all-t & $405(1.56)$ & 410 (417) \\
\hline
\end{tabular}

${ }^{a}$ See Table 7 for calculations on more $\alpha n$ 's and by different methods. Here, ZINDO is used for theory. All are this work. ${ }^{b}$ See Table 7 for other transitions for these and other $\alpha$-oligothiophenes. ${ }^{c}$ Values in methylcyclohexane. Values in dioxane are in parentheses. ${ }^{d}$ In all cases except STO3G there were finite values of twist for the $\mathrm{S}-\mathrm{C}-\mathrm{C}-\mathrm{S}$ dihedral angle while for STO $3 \mathrm{G}$ there was no twist (totally planar).

geometry (no twisting) for the all-trans conformer, as well as for the all-cis one (interestingly enough, essentially all the calculation methods give near planar geometry for the all-cis conformer). Some X-ray data on $\alpha 4$ and $\alpha 6^{45}$ give evidence for an all-trans, nearly planar $\left(0-10^{\circ}\right.$ twist $)$ geometry. There would be no reason to believe $\alpha 5$ should be different.

In Table 6 we present the lowest transition energy predicted for the all-trans and all-cis conformers of $\alpha 2, \alpha 3$, and $\alpha 5$ and a comparison with experimental data. It can be clearly seen that for $\alpha 5$, all three methods of geometry optimization show that the all-trans conformer spectral data are very much closer to experimental results than the all-cis. The best agreement is from STO3G, and recall that this was the one that gave no twisting of the inter-ring bond. We believe this is perhaps the strongest evidence that $\alpha 5$ is all-trans with very little inter-ring twisting, and a similar situation should be true for $\alpha 3$.

Table 7 gives experimental data for the discernible transitions in $\alpha 1-\alpha 7$, extinction coefficients, and theoretical predictions for a number of $\alpha$-oligothiophenes.

\section{Discussion}

State Order. One of the most important questions for the $\alpha$-oligothiophenes (hereafter denoted $\alpha n$ ) concerns the state order: does the state order change with increasing $n$, and if so, when?

Based on earlier spectroscopic date of $\alpha, \omega$-dithienylpolyenes, calculations, and some extrapolated data, the (2) ${ }^{1} \mathrm{Ag}$ state was assigned below the $(1)^{1} \mathrm{Bu}$ for oligothiophenes of $n=6$ and greater. ${ }^{7}$ For $\alpha 2$, two-photon spectroscopy ${ }^{5}$ has located the ${ }^{1} \mathrm{Ag}$ above the ${ }^{1} \mathrm{Bu}$. Theoretically, it has been predicted ${ }^{8}$ that the ${ }^{1} \mathrm{Ag}$ state would be below the ${ }^{1} \mathrm{Bu}$ state for $\alpha 2-\alpha 4$ with crossing between $\alpha 4$ and $\alpha 5$. However, reduction in the degree of $\mathrm{CI}$ (with no rational justification) now resulted in the ${ }^{1} \mathrm{Bu}$ state being lowest from $\alpha 2$ to $\alpha 7$ (others predicted ${ }^{1} \mathrm{Ag}$ to be lowest for $\alpha 6$ and longer chain). Also, more recent calculations ${ }^{9}$ for $\alpha 1-\alpha 4$ predicted that the ${ }^{1} \mathrm{Ag}$ state ( 0 level) would be below the ${ }^{1} \mathrm{Bu}$ state $\left(0\right.$ level). They believed the ${ }^{1} \mathrm{Ag}$ state observed was the $(3)^{1} \mathrm{Ag}$ state. Multi-CI ab initio calculations on 
TABLE 7: Experimental (with $\epsilon$ ) and Theoretical ${ }^{a}$ Energies (with $f$ ) for All-Trans Conformers of Some $\alpha$-Oligothiophenes

\begin{tabular}{|c|c|c|c|c|}
\hline \multirow[b]{2}{*}{ cpd } & \multicolumn{2}{|c|}{$\operatorname{exptl}^{b}$} & \multicolumn{2}{|c|}{ theoretical } \\
\hline & $\begin{array}{c}\lambda \\
(\mathrm{nm})\end{array}$ & $\begin{array}{c}\epsilon \\
\left(\mathrm{M}^{-1} \mathrm{~cm}^{-3}\right)\end{array}$ & $\begin{array}{c}\lambda \\
(\mathrm{nm})\end{array}$ & $f$ \\
\hline \multirow[t]{3}{*}{ thiophene } & 232 & 8340 & 227 & $0.26(\mathrm{PPP})^{d}$ \\
\hline & & & 211 & 0.27 \\
\hline & & & 170,169 & 1.5 \\
\hline \multirow[t]{3}{*}{ di- } & $303(302)^{c}$ & 12440 & 311 & $0.83(\mathrm{PPP})^{d}$ \\
\hline & 247 & 6200 & 257 & 0.39 \\
\hline & 205 & & 204 & 0.19 \\
\hline \multirow{5}{*}{ tri- } & $354(350)^{c}$ & 22100 & 342 & $1.40(\mathrm{PPP})^{d}$ \\
\hline & & & 276 & 0.02 \\
\hline & 252 & 8600 & 250,245 & 0.24 \\
\hline & 221 & & 234 & 0.15 \\
\hline & 205 & & 222 & 0.22 \\
\hline \multirow[t]{4}{*}{ quater- } & $391(386)^{c}$ & 31560 & & \\
\hline & $308(\mathrm{sh})$ & 4300 & & \\
\hline & 252 & 10700 & & \\
\hline & 205 & & & \\
\hline \multirow[t]{5}{*}{ quinque- } & $\begin{array}{l}417(410)^{c} \\
\sim 340(\mathrm{sh})\end{array}$ & 42650 & 405 & 1.56 (ZINDO) \\
\hline & 290 & 7300 & 286 & 0.12 \\
\hline & 256 & 12700 & 256,260 & 0.06 \\
\hline & $207-214$ & & 236 & 0.10 \\
\hline & & & 196 & 1.71 \\
\hline \multirow[t]{3}{*}{ sexi- } & 436 & 47910 & & \\
\hline & 314 & & & \\
\hline & 259 & & & \\
\hline \multirow[t]{6}{*}{ septa- } & $441(433)^{c}$ & 50460 & & \\
\hline & $361(\mathrm{sh})$ & & & \\
\hline & 325 & 10900 & & \\
\hline & $282,298(\mathrm{sh})$ & & & \\
\hline & 256 & 18200 & & \\
\hline & 205 & & & \\
\hline
\end{tabular}

${ }^{a}$ PPP model with geometries from an MMX (force field) approximation (Serena Software, Bloomington IN) for thiophene, bithiophene, and trithiophene. For quinquethiophene data are from ZINDO using geometry obtained via STO 3G. ${ }^{b}$ In ethanol for thiophene, all others in dioxane. See Experimental Section for methodology for determining $\epsilon{ }^{c}$ Values in parentheses are for the compound in methylcyclohexane. ${ }^{d}$ Parameters for PPP calculation were $I_{\mathrm{P}}(\mathrm{C})=11.20, \gamma(\mathrm{C})=11.10$, $I_{\mathrm{P}}(\mathrm{S})=17.20, \gamma(\mathrm{S})=10.00, \beta_{\mathrm{CS}}=-1.8, \beta_{\mathrm{CC}}=-2.4$.

thiophene $(\alpha 1)$ place the $(2)^{1} \mathrm{~A}_{1}$ state below the ${ }^{1} \mathrm{~B}_{2} \cdot{ }^{46}$ More recently, $a b$ initio calculations ${ }^{11}$ (multi-CI) for $\alpha 2$ give a ${ }^{1} \mathrm{Bu}$ state below the ${ }^{1} \mathrm{Ag}$ state and, in fact, predicts two ${ }^{1} \mathrm{Bu}$ states below the lowest ${ }^{1} \mathrm{Ag}$. Very recent calculations ${ }^{3}$ used CNDO/S both with single and single plus double CI to calculate transition energies and state orders. Good results compared with experimental results were obtained with single CI but not with single plus double CI. This problem with double CI and of rationalizing the results when ignoring double CI has been a major problem (see refs 3,7 , and 8 ).

From our experimental data the $k_{\mathrm{F}}^{0}$ values in benzene (Table 1) for six oligothiophenes, $\alpha 2-\alpha 7$, show that the $k_{\mathrm{F}}^{0}$ is remarkable constant. Furthermore, $k_{\mathrm{F}}^{0}$ is essentially constant in all four additional solvents in Table 2, especially for $\alpha 4-\alpha 7$. Moreover, the $k_{\mathrm{F}}^{0}$ values for all the $\alpha$-oligothiophenes in all the solvents only vary from $(0.28-0.45) \times 10^{9} \mathrm{~s}^{-1}$, most values being in a narrower range still (others ${ }^{3}$ found a range of $(0.25-$ $0.33) \times 10^{9} \mathrm{~s}^{-1}$ in dioxane). These correspond to $\tau_{\mathrm{F}}^{0}$ values of 2.3-3.5 ns, which are clearly typical of emission lifetimes from allowed $\pi, \pi^{*}$ states. Also, as given earlier ${ }^{1}, k_{\mathrm{F}}^{0}$ values calculated based on the Strickler-Berg (SB) equation over the first absorption band for $\alpha 2-\alpha 5$ and $\alpha 7$ agreed within $0-14 \%$ (only one case was as large as $14 \%$; others were less than $7 \%$ ) of those from experiment. The result of all of these considerations provides experimental convincing evidence that the lowest excited state for $\alpha 2-\alpha 7$ is of allowed character and is $(1)^{1} B u$ and not $(2)^{1} A g$. This was noted earlier ${ }^{1}$ for $\alpha 2-\alpha 5$ and $\alpha 7$ in benzene, but we can now include $\alpha 6$ as well and, moreover, safely conclude that this order is independent of the solvent for $\alpha 2-\alpha 7$

Relative to the question of whether the $(1)^{1} \mathrm{Bu}$ and $(2)^{1} \mathrm{Ag}$ state cross and, if so, when, it is difficult to answer when, but surely they will cross, since the two states are getting closer as $n$ increases (for $\alpha$ ). Earlier, on the basis of only two data points for extrapolation of the $(2)^{1} \mathrm{Ag}$ energy, we believed crossing might occur between $n=9$ and $n=11$. However, the $(2)^{1} \mathrm{Ag}$ data were from different phases of different $\alpha n$ 's, so we no longer believe the crossing will occur at these low $n$ values. Others $^{3}$ also note that the states will cross, but based on some CNDO/S and FEMO models, they predicted crossing would be beyond $n=50$.

Temperature Effect on Absorption and Ground-State Geometry. Recall earlier in the Results section that we noted the very significant red shift $\left(\sim 1600 \mathrm{~cm}^{-1}\right)$ of the absorption maxima of $\alpha 2-\alpha 7$ upon going from 298 to $77 \mathrm{~K}$ (see Figure 3 and ref 1 ). If, however, we look at the $0-0$ band (as determined by the overlap of absorption and fluorescence spectra at 298 and $77 \mathrm{~K}$ ), the shift is greatly reduced to only $\sim 200 \mathrm{~cm}^{-1}$ for $\alpha 2$ and $\alpha 3, \sim 300 \mathrm{~cm}^{-1}$ for $\alpha 4$, and $\sim 400 \mathrm{~cm}^{-1}$ for $\alpha 5$ and $\alpha 7$. We interpret the absorption results to mean that as the temperature is lowered, there is a greater average planarity among existing conformers; said in another way, the virtual molecule at $77 \mathrm{~K}$ is more planar than one at $298 \mathrm{~K}$. That is, the potential energy minimum in the ground state becomes more vertically aligned to that of the excited state such that the vertical transition now is to a lower vibronic level of the excited state, yet the actual state energy difference (0-0's) has changed relatively little. Usually, the red shift seen in the absorption upon going to low temperature is ascribed to increased planarity of the ground state with subsequent greater conjugation, lengthening of the $\pi$-electron path, and decreased energy gap between $S_{0}$ and $S_{1}$. However, based on our spectroscopic data as described above, this is not what principally occurs. It does appear that there is some small effect on relative state energies for $\alpha 5-\alpha 7$ and perhaps for $\alpha 4$.

There are clear indications that $\alpha 2$ is twisted in both the cis and trans forms, that they coexist, and that the trans is more stable than the cis. Furthermore, the absorption (and fluorescence) of $\alpha 2$ undergo the most dramatic increase in vibrational resolution as a function of temperature compared with all other $\alpha n$ 's (see Figure 5 and ref 1). However, the red shift of the absorption maxima at low temperature is essentially the same as for $\alpha 3-\alpha 5\left(\sim 1600 \mathrm{~cm}^{-1}\right)$ and for the others, while the fluorescence maximum shows essentially no shift $\left(\leq 200 \mathrm{~cm}^{-1}\right)$. We interpret all the absorption results to mean that for $\alpha 2$ the ground state becomes more planar, as it is for all $\alpha$ n's, and a greater population of a more singular conformer exists for $\alpha 2$ compared to other $\alpha n$ 's at low temperature.

Temperature Effect on Fluorescence and Excited-State Geometry. A negligible shift of the maximum of fluorescence $\left(100-200 \mathrm{~cm}^{-1}\right)$ as well as the $0-0$ band occurs upon lowering the temperature. We believe that based on the Franck-Condon forbidden shape of the first absorption band, an obvious change in geometry has occurred. This could be consistent with a quinoidal-like contribution to the structure of the excited state-we will support this belief more fully shortly. At room temperature, solvent shell relaxation around the now quinoidallike excited state can occur, so emission occurs from the equilibrium excited state to a twisted ground state. However, at $77 \mathrm{~K}$ in a rigid matrix environment solvent shell relaxation 
TABLE 8: Atomic Charges and Dipole Moments in the Ground and Excited States ${ }^{a}$

\begin{tabular}{|c|c|c|c|c|c|c|}
\hline cpd & atom & GS charge & $\mu(\mathrm{D})$ & ES $\left(S_{1}\right)$ charge & $\mu(\mathrm{D})$ & ES $\left(\mathrm{T}_{1}\right)$ charge \\
\hline$\alpha 1$ & $\begin{array}{l}\text { S5 } \\
\text { C1, C2 }(\alpha-C) \\
\text { C3, C4 }(\beta-C)\end{array}$ & $\begin{array}{l}+0.02 \\
-0.14 \\
-0.03\end{array}$ & 1.84 & $\begin{array}{l}-0.22 \\
+0.03 \\
-0.01\end{array}$ & 2.52 & \\
\hline$\alpha 2$ & $\begin{array}{l}\text { S5, S13 } \\
\text { C4, C11 }(\alpha-\mathrm{C}) \\
\text { C1, C10 }(\beta-\mathrm{C}) \\
\text { C2, C9 }(\beta-\mathrm{C}) \\
\text { C3, C12 }(\alpha-\mathrm{C}, \text { bonded }) \\
\text { H7, H14 }\end{array}$ & $\begin{array}{l}+0.004(+0.58) \\
-0.14(-0.45) \\
-0.03(-0.15) \\
-0.04(-0.16) \\
-0.035(-0.30) \\
+0.075\end{array}$ & 0.007 & $\begin{array}{l}-0.15(+0.38) \\
-0.01(-0.35) \\
+0.015(-0.09) \\
-0.025(-0.11) \\
+0.05(-0.29) \\
+0.04\end{array}$ & 0.009 & $\begin{array}{l}(+0.53) \\
(-0.47) \\
(-0.10) \\
(-0.20) \\
(-0.24)\end{array}$ \\
\hline$\alpha 3$ & $\begin{array}{l}\text { S5, S20 } \\
\text { C4, C18 }(\alpha-C) \\
\text { C1, C17 }(\beta-C) \\
\text { C2, C8 }(\beta-C) \\
\text { C3, C19 }(\alpha-C, \text { bonded }) \\
\text { S14 } \\
\text { C12, C13 }(\alpha-C, \text { bonded }) \\
\text { C10, C11 }(\beta-C) \\
\text { H7 } \\
\text { H15 } \\
\text { H16 } \\
\text { H21 }\end{array}$ & $\begin{array}{l}+0.004 \\
-0.14 \\
-0.03 \\
-0.04 \\
-0.035 \\
+0.001 \\
-0.04 \\
-0.04 \\
+0.074 \\
+0.077 \\
+0.076 \\
+0.074\end{array}$ & 1.84 & $\begin{array}{l}-0.14 \\
-0.02 \\
+0.01 \\
-0.014 \\
+0.04 \\
-0.16 \\
+0.04 \\
-0.005 \\
+0.04 \\
+0.04 \\
+0.04 \\
+0.04\end{array}$ & 1.77 & \\
\hline$\alpha 5$ & $\begin{array}{l}\text { S26, S34 } \\
\text { C25, C19, C18, C11, } \\
\text { C12, C4, C3, C32c } \\
\text { C16, C31 }(\beta-\mathrm{C}) \\
\text { C23, C30 }(\beta-\mathrm{C}) \\
\text { C24, C33 }(\alpha-\mathrm{C}) \\
\text { H27, H22, H21, H15, } \\
\text { H14, H7, H6, H36 } \\
\text { S10, S13, S5 } \\
\text { C17, C1 }(\beta-\mathrm{C}) \\
\text { C10, C9, C } 8, \text { C2 }(\beta-\mathrm{C})\end{array}$ & $\begin{array}{l}+0.02 \\
-0.05^{c} \\
-0.04 \\
-0.03 \\
-0.15 \\
+0.075^{b} \\
+0.02 \\
-0.035 \\
-0.04\end{array}$ & 2.11 & $\begin{array}{l}-0.12 \\
+0.03^{c} \\
+0.00 \\
+0.01 \\
-0.04 \\
+0.04 \\
\\
-0.14 \\
+0.005 \\
-0.003\end{array}$ & 2.16 & \\
\hline
\end{tabular}

${ }^{a}$ See Figure 10 for structures and numbering of atoms. Charges are formal charges calculated by the ZINDO approximation, and for $\alpha 2$, the numbers in parentheses are those calculated using the AMPAC approximation using the AM1 Hamiltonian (full SCF and configuration interaction). ${ }^{b}$ This represents an average of values between 0.074 and $0.077 .{ }^{c} \alpha-\mathrm{C}$, bonded.

cannot occur and emission is from the Franck-Condon excited state to a "planar" ground state. It is this combination of the different circumstances that results in essentially no shifting of fluorescence bands between 298 and $77 \mathrm{~K}$. The above interpretations regarding absorption (previous section) and fluorescence behavior are supported in two major ways. One source of support regards the spectroscopic studies of bithiophene $(\alpha 2)$ in a supersonic jet ${ }^{42}$ where there is a clear indication that in $S_{1}$ the $\alpha 2$ is trans planar with a single minimum and that a deep, steep potential well exists. However, in $\mathrm{S}_{0}$, a much broader potential well exists (and in fact there is a double minimum, and equivalent twisted forms exist). There is no reason to believe a parallel situation is not true in general for all of the $\alpha$ 's albeit the ground state may not have multiminima but that the excited state has a higher bond order for the interring bonds and is planar quinoidal-like compared to the ground state. A second source of support involves a consideration of changes in atomic charges between the $S_{0}$ and $S_{1}$ state (as well as the $\mathrm{T}_{1}$ state, which we will need to consider shortly). Table 8 gives the atomic charge and dipole moments in the ground state and $S_{1}$ state for all atoms in $\alpha 1-\alpha 3$ and $\alpha 5$ and atomic charges in $\mathrm{T}_{1}$ for $\alpha 2$, where Figure 10 gives the appropriate structures and numbering for these four molecules. Note that in all cases, there is large change in charge on sulfur and the $\alpha$-carbon (non inter-ring bonded) between $S_{0}$ and $S_{1}$ (Table 8). In the case of sulfur, the change involves a large addition of negative charge $[\sim 0.14-0.24 e]$ while for $\alpha$-carbons that are
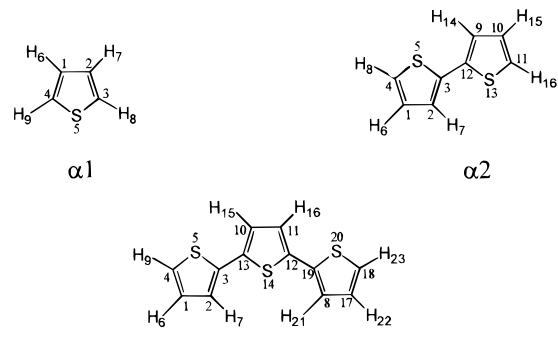

$\alpha 3$

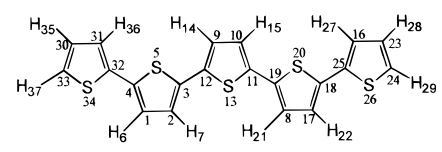

$\alpha 5$

Figure 10. Numbering of atoms of various oligothiophenes for reference to atomic charges given in Table 8 .

non-inter-ring bonded, there is a quite large subtraction of negative charge $(\sim 0.12-0.19 e)$. The bonded $\alpha$-carbons (to another $\alpha$-carbon) still show subtraction of negative charge but smaller in magnitude $(\sim 0.07 e)$. One obvious important implication of this is that the lowest energy transition (longest wavelength one) now clearly established to be

$$
{ }^{1} \mathrm{~A} \rightarrow(1)^{1} \mathrm{Bu}
$$


has some charge transfer character. Secondly, with such large changes in atomic charges there must be an accompanying change in the geometry of the excited singlet state (as previously noted, based simply on the Franck-Condon forbidden shape of the first transition). A quinoidal-like structure is compatible with these charge changes and totally in harmony with the change in the ground and excited singlet state potential energy curves along the inter-ring $\alpha \mathrm{C}-\alpha \mathrm{C}$ torsional coordinate found in supersonic jet experiments ${ }^{42}$ where for $\alpha 2$, the inter-ring $\alpha \mathrm{C}-$ $\alpha \mathrm{C}$ bond was clearly much more double bonded in $\mathrm{S}_{1}$ compared to $\mathrm{S}_{0}$.

In addition to studying the effect of temperature on absorption and fluorescence spectral maxima and $0-0$ origins, we examined the dependence of $\phi_{\mathrm{F}}$ on temperature. In Table 2 for ethanol as the solvent, it is interesting to note that $\phi_{\mathrm{F}}$ for $\alpha 2$ and $\alpha 3$ clearly increase from 298 to $77 \mathrm{~K}$ by 2.8 -fold and 2 -fold, respectively. However, there is essentially no change in $\phi_{\mathrm{F}}$ for the other $\alpha$ n's $(\alpha 4-\alpha 7)$. Others ${ }^{35}$ also found a very similar change for $\alpha 3$ and, furthermore, by assuming that the only temperature dependent process was nonradiative, calculated an activation energy of $\sim 1.2 \mathrm{kcal} / \mathrm{mol}$. We also examined the effect of temperature on $\phi_{\mathrm{T}}$ of $\alpha 3$ and found little or no change over the range $290-140 \mathrm{~K}$. Others ${ }^{35}$ believed that the activation energy was for $\mathrm{S}_{1} \rightarrow \mathrm{T}_{n}$ and that about $55 \%$ of the $\phi_{\mathrm{T}}$ arose from this path.

Variation of Rate Constants. Geometry and Charge Transfer Mixing. First of all, recall that based on the $k_{\mathrm{F}}^{0}$ and $\tau_{\mathrm{F}}^{0}$ values in the five solvents (Tables 1 and 2) and the correlation with those determined by the Strickler-Berg equation, the evidence is totally convincing that the ${ }^{1} \mathrm{Bu}$ state is the lowest for all $\alpha n$ 's from $\alpha 2$ to $\alpha 7$, and moreover, this order is solvent independent.

In general, the $k_{\mathrm{F}}^{0}$ is quite constant $(\sim 20 \%)$ as a function of the number of rings $(\alpha 2-\alpha 7)$ and solvent (except perhaps benzene where it is sometimes greater) while $k_{\text {ISC }}$ undergoes at least a 20-fold (vs $20 \%$ for $k_{\mathrm{F}}^{0}$ ) decrease between $\alpha 2$ and $\alpha 5$ and a leveling of $k_{\mathrm{ISC}}$ occurs from $\alpha 5$ to $\alpha 7$. The trend of $k_{\mathrm{IC}}$ is not so definitive because as noted earlier $\phi_{\mathrm{T}}+\phi_{\mathrm{F}} \approx 1$ and because of the potentially significant error in $\phi_{\mathrm{T}}( \pm 15 \%)$; the difference term $\left(1-\phi_{\mathrm{T}}-\phi_{\mathrm{F}}\right)$ required to calculate $k_{\mathrm{IC}}$ can generate notable error in the $k_{\mathrm{IC}}$. However, it is clear that it is significantly smaller than $k_{\text {ISC }}$ (Tables 1 and 2). Furthermore, there is a clear, progressive decrease in $k_{\mathrm{NR}}$ (Tables 1 and 2) from $\alpha 2$ to $\alpha 5$ and than a leveling from $\alpha 5$ to $\alpha 7$ (as for $k_{\mathrm{ISC}}$ ). A similar trend is apparent for $k_{\text {IC }}$ in dioxane (Table 2). On the basis of the relationship of $k_{\mathrm{ISC}}, k_{\mathrm{F}}$, and $k_{\mathrm{IC}}$ as well as the fact that $\phi_{\mathrm{F}}+\phi_{\mathrm{T}}$ generally is $>0.92, k_{\mathrm{IC}}$ is very small, and therefore, internal conversion from $S_{1}$ is small or negligible $\left(\phi_{\text {IC }}\right.$ $\approx 0.1-0.0$ ).

We believe that the preceding facts can be understood by a combination of circumstances. There is evidence from two sources that the first excited singlet state has a quinoidal-like character that would account for the very small internal conversion observed, since there would be very little torsional mode coupling (around the inter-ring bond) to the ground state. There is obviously significant spin-orbit coupling that has to be due in part to the heavy atom effect ${ }^{47}$ of the sulfur. Nonetheless, $\phi_{\mathrm{T}}$ and $k_{\mathrm{ISC}}$ show a progressive decrease from $\alpha 2$ to $\alpha 5$ and then a leveling off (while $\phi_{\mathrm{F}}$ shows a progressive increase from $\alpha 2$ to $\alpha 5$ and then a leveling off from $\alpha 2$ to $\alpha 7$ ). We believe that most of the spin-orbit coupling is not due to the classical heavy atom effect ${ }^{47}$ but that this coupling is mediated by charge transfer (CT) mixing involving matrix elements of the type

$$
\left\langle{ }^{1} \psi_{\mathrm{CT}}\left|\mathrm{H}^{\prime}\right|^{3} \psi_{i}\right\rangle
$$

where $\mathrm{H}^{\prime}$ is the spin-orbit (coupling) operator and contains the atomic spin-orbit coupling factor $\zeta$ for sulfur, which is large, $363 \mathrm{~cm}^{-1}$. Remember that the decrease in $\phi_{\mathrm{T}}$ (and increase in $\left.\phi_{\mathrm{F}}\right)$ is not due to some significant increase in $k_{\mathrm{F}}^{0}$, which is essentially constant, or $k_{\mathrm{IC}}$ ( $k_{\mathrm{NR}}$ as the number of rings increases) but is due to a real decrease in $k_{\text {ISC }}$ (Tables 1 and 2). It is clear that as the number of rings is increased, a like number of heavy atom sulfur centers is also increased. Yet, $k_{\mathrm{ISC}}$ (and $f_{\mathrm{T}}$ ) still decreases. We believe this occurs because of a decrease in the magnitude of the matrix elements described above, resulting from a decreased charge transfer mixing of ${ }^{1} \psi_{\mathrm{CT}}$ and ${ }^{3} \psi_{i}$ as the number of rings increases. This may result from the smaller overlap of the electron donor-electron acceptor molecular orbitals because of the more spread-out nature of the molecular orbitals proceeding from $\alpha 2$ to $\alpha 5$, which becomes essentially constant from $\alpha 5$ to $\alpha 7$.

Absence of Phosphorescence and Triplet State Lifetimes. Significant inter-ring bond torsional (twisting) coupling to the ground state could occur, resulting in a large radiationless rate constant for $T_{1} \rightarrow S_{0}$ with a quenching of phosphorescence emission. Recall that the lifetime of the triplet state undergoes a substantial decrease from $\alpha 2$ to $\alpha 7$ in all solvents (see Tables 1 and 2) by 5- to 10-fold. If the lifetimes are considered to be essentially the radiationless lifetimes, then the radiationless intersystem crossing $T_{1} m \rightarrow S_{0}$ would strongly dominate and phosphorescence would be "absent". In such a case, the lifetimes would be expected to decrease because of the decrease in the $T_{1}-S_{0}$ energy gap $(\alpha 2-\alpha 7)$ resulting in a significant increase in the Franck-Condon overlap integrals between $\mathrm{T}_{1}$ and $\mathrm{S}_{0}$ upon which the radiationless rate constants depend. ${ }^{47}$

We make one additional observation regarding $\alpha 2$. Some two-photon photoionization experiments ${ }^{37}$ assign the final state as a triplet state (related to the dominant photoionization channel). Earlier in the Results section, we compared some triplet lifetimes and in the particular case of $\alpha 2$, we determined $\tau_{\mathrm{T}}$ to be 100-146 $\mu \mathrm{s}$ (solvent dependent) at room temperature in a fluid solution (Tables 1 and 2). The $\tau_{\mathrm{T}}$ given for the triplet state of $\alpha 2$ in the supersonic jet experiment above ${ }^{37}$ was 550 ns. This triplet lifetime seems to be extraordinarily short for a molecule in a nearly isolated condition presumably without (or nearly without) external intermolecular interaction (at the usually expected chamber background pressures) and where, although it is not strictly possible to define the temperature, a temperature on the order of $10 \mathrm{~K}$ seems reasonable. The lifetime is $\sim 225$ fold shorter than our triplet lifetimes in a variety of solvents that were not vacuum degassed (but $\mathrm{N}_{2}$ bubbled) at room temperature (vs temperature on the order of $10 \mathrm{~K}$ for the supersonic jet experiment), where intermolecular interactions must be more plentiful than in the supersonic jet experiment. These considerations make us very doubtful of the assignment of the $T_{1}$ triplet state (of $\alpha 2$ ) as the state to be associated with the 550 ns lifetime.

Recall that thiophene itself, $\alpha 1$, did show a weak phosphorescence but "no" fluorescence $\left(\phi_{\mathrm{F}} \leq 5 \times 10^{-4}\right)$. We do not know whether phosphorescence is weak because $\phi_{\mathrm{T}}$ is low $\left(\phi_{\mathrm{IC}}\right.$ is high) or because the nonradiative process from $\mathrm{T}_{1}$ is high. Nonetheless, phosphorescence does exist at a level greater than any other $\alpha n$ so far examined $(\alpha 2-\alpha 4, \alpha 6)$. Given that it would be reasonable to expect $\phi_{\mathrm{T}}$ to be quite high because of matrix elements of the type described in the previous section above (thiophene also has a large amount of charge transfer going from $\mathrm{S}_{0} \rightarrow \mathrm{S}_{1}$, Table 8), we would expect $\phi_{\mathrm{P}}$ to be high. Thus, the reason phosphorescence is weak is very likely because of a 
highly efficient nonradiative process out of $\mathrm{T}_{1}$. This is supported by the short $\tau_{\mathrm{T}}(1 \mu \mathrm{s})$ compared to those of all other $\alpha$ 's.

Singlet Oxygen Formation by Energy Transfer. In Table 1 , it can be seen that the $\phi_{\Delta}$ decreases with an increase in the number of rings. Note that for $\alpha 3$, the efficiency of triplet energy transfer to produce ${ }^{1} \mathrm{O}_{2}, S_{\Delta}=\phi_{\Delta} / \phi_{\mathrm{T}}$, is 0.85 while for $\alpha 2, \alpha 4$, and $\alpha 5$ it is near 1 . These results clearly indicate energy transfer is highly efficient from many oligothiophenes to ground state oxygen. Although we are not totally confident about the $\phi_{\Delta}$ for $\alpha 7$ ( or $\phi_{\mathrm{T}}$ ), it appears to be in the $0.2-0.3$ range, giving a $S_{\Delta}$ that is relatively low $(\sim 0.5)$. This may be because of the presence of two $n$-butyl groups preventing efficient encounter transfer and the fact that the triplet is expected to be quite low for $\alpha 7\left(9-12000 \mathrm{~cm}^{-1}\right)$. The situation for $\alpha 6$ is unclear, although based on $\phi_{\mathrm{T}}$ data in two solvents for $\alpha 5$ and one for $\alpha 7$, it seems that $\phi_{\mathrm{T}}$ for $\alpha 6$ should be in the 0.5 area. If true, the $S_{\Delta}$ is definitely lower than for the others except possibly for $\alpha 7$, based on the literature value (Table 1) of $0.36^{4}$ for $\phi_{\Delta}$. There are several literature values for the $\phi_{\Delta}$ of $\alpha 3$ : $0.15,{ }^{26}$ $\sim 0.8,{ }^{1,4} \sim 0.8,{ }^{48} 0.73^{30}$ (which was believed to be more accurate than an earlier 0.86 value determined by some of the same authors), and 0.6 or 0.86 depending on the technique used ${ }^{49}$ compared with our values of 0.81 in benzene and 0.74 in acetonitrile. We believe the $0.15^{26}$ value is very probably incorrect for $\alpha 3$. On the basis of all the data available, we believe $\phi_{\Delta}$ for $\alpha 3$ is $0.75 \pm 0.05$.

The $\phi_{\Delta}$ values of other $\alpha n$ 's have been given to be ${ }^{4} \sim 0.7$ for $\alpha 4, \sim 0.5$ for $\alpha 5$, and $\sim 0.36$ for $\alpha 6$, where both $\alpha 4$ and $\alpha 5$ (as well as $\alpha 3$ ) are close to our values (see Table 1). Elsewhere, ${ }^{26}$ a value of 0.24 has been determined for $\alpha 4$. We believe this value of $0.24^{26}$ is quite probably incorrect for $\alpha 4$ based on our value of $\phi_{\mathrm{T}}$ of $\sim 0.7$. Furthermore, we and others ${ }^{4}$ find a value of $\sim 0.7$ for $\phi_{\Delta}$ of $\alpha 4$ and, of course, $\phi_{\Delta}$ cannot be greater than $\phi_{\mathrm{T}}$ as it would be if $\phi_{\mathrm{T}}=0.24 .^{26}$ The $\phi_{\Delta}$ values of some substituted bithiophenes (acetylenic, olefinic) are known, ${ }^{50}$ and these all seem to be quite low, $\sim 0.05$, compared to what we obtain for bithiophene itself, $\sim 0.97$ (Tables 1 and 2).

Comparison of Solution and Film Data. Recall that there is great interest in oligothiophenes regarding their application in nonlinear optical charge storage and molecular electronics devices. In most of these applications, the solid phase is the phase of most interest. However, because of film quality, thickness, homogeniety, and crystallinity, as well as the ambient conditions, the absorption, fluorescence, and other photophysical properties truly intrinsic to a particular $\alpha n$ are not well defined. Therefore, a study as carried out here can provide intrinsic properties at the molecular level, and these can then be used as a reference to better understand and interpret solid state absorption and photophysical results.

For films of the oligothiophenes, assignment of the lowest excited state varies. Studies of films and solutions ${ }^{4}$ showed that the $\phi_{\mathrm{F}}$ of ultrathin and thin films were considerably less in general than those in solutions $\left(10-10^{3}\right.$ times) and that the absorption maximum was strongly blue-shifted ${ }^{51}$ (although absorption could continue to near the same onset). Also, fluorescence decay curves of films are strongly nonexponential, ${ }^{51}$ in contrast to our work in solution. This indicates considerable inhomogeniety in the surroundings of the oligomers. The lowest excited state was assigned as $(2)^{1} \mathrm{Ag}$ for all oligothiophenes including $\alpha 2-\alpha 8^{3}$. However, although this assignment for all was repeated again, ${ }^{51}$ there was some question about how unambiguous it was. Others ${ }^{45}$ have assigned the lowest excited state for films $\alpha 6$ as $(1)^{1} \mathrm{Bu}$, which was noted to be some $900 \mathrm{~cm}^{-1}$ below the (2) ${ }^{1} \mathrm{Ag}$ (comparing lowest exciton levels of each exciton band). The fluorescence (radiative) lifetime was given as shorter than 600 ps. Recall that our work in solutions clearly defines the lowest excited state of the oligomers $\alpha 2-\alpha 7$ as $(1)^{1} \mathrm{Bu}$.

Absorption data for a single crystal of $\alpha 6$ indicated that the maximum was red-shifted compared to the solution data, ${ }^{45}$ whereas for oriented thin films, it was blue-shifted. ${ }^{51}$ Other studies on thin films of $\alpha 6$ found the excited singlet state lifetime to be $\sim 40$ ps and a triplet-triplet (sharp) absorption near 790 $\mathrm{nm}$ with a lifetime of $4 \mathrm{~ns}$ for the triplet state. ${ }^{52}$ The singlet lifetime is very short compared to that in solution (24-fold), but in general $\phi_{\mathrm{F}}$ values are also much lower, so some quenching mode exists. The short $4 \mathrm{~ns}$ triplet lifetime is at least partially explained by the presence of air.

Thin polycrystalline films of $\alpha 4-\alpha 6$ showed transient absorptions that were assigned as $\mathrm{T}_{1} \rightarrow \mathrm{T}_{n}$ absorption. ${ }^{53}$ Lifetimes of $310 \mathrm{~ns}(\alpha 6), 550 \mathrm{~ns}(\alpha 5)$, and $1.3 \mu \mathrm{s}(\alpha 4)$ were reported (presumably in the presence of air). These are 4050 times shorter than ours in degassed solutions (see Tables 1 and 2). In a crystalline film (at 5-10 Torr) the $\tau_{\mathrm{T}}$ of $\alpha 6(\sim 170$ $\mu \mathrm{s})$ is very much longer than that found above $(4 \mathrm{~ns})^{52}$ and even much longer than in degassed solutions $(\sim 17 \mu$ s, Table 1$)$. The lifetimes of a film of $\alpha 7(\sim 100 \mu \mathrm{s})$ is also much longer than that in degassed solution (21 $\mu \mathrm{s}$, Table 1).

\section{Summary/Conclusions}

1. Based on the $k_{\mathrm{F}}^{0}$ of $\alpha 2-\alpha 7$ in any and all five solvents, the lowest excited singlet state is $(1)^{1} \mathrm{Bu}\left(\pi, \pi^{*}\right)$.

2. There is a significant shift $\left(\sim 1600 \mathrm{~cm}^{-1}\right)$ of the absorption maxima of $\alpha 2-\alpha 7$ upon going from 295 to $77 \mathrm{~K}$, but much smaller shifts occur for the $0-0$ band $\left(200-400 \mathrm{~cm}^{-1}\right)$. We interpret this to be largely due to an increase in the overall planarity of the ground state among the existing conformers (or virtual molecule) at $77 \mathrm{~K}$. That is, the potential energy minima of $S_{0}$ and $S_{1}$ are more vertically aligned such that the vertical transition is to a lower vibronic level of $S_{1}$ (but the actual state energy difference is little changed).

3. Significant changes in atomic charge occur on sulfur and the $\alpha$-carbons of all $\alpha$ 's when comparing $\mathrm{S}_{1}$ to $\mathrm{S}_{0}$ (and $\mathrm{T}_{1}$ ) but not when comparing $T_{1}$ to $S_{0}(\alpha 2)$. These are compatible with geometry changes between $S_{0}$ and $S_{1}$ but not between $S_{0}$ and $\mathrm{T}_{1}$ (also see items 4-6, below).

4. On the basis of absorption spectral shape, charge density calculations of the ground and first excited states, and existing literature, ${ }^{42}$ we believe the excited singlet state, $S_{1}$, has a quinoidal-like form and therefore is essentially planar while the ground state is twisted for all $\alpha$ 's.

5. There is almost no shift $\left(100-200 \mathrm{~cm}^{-1}\right)$ of the fluorescence maxima of $\alpha 2-\alpha 7$ (in contrast to absorption). We interpret this as the result of a combination of circumstances, including a quinoidal-like planar $S_{1}$ state, a twisted ground state, and the presence of (at $295 \mathrm{~K}$ ) or absence of (at $77 \mathrm{~K}$ ) of solvent reorientation.

6. The small magnitude of $\phi_{\text {IC }}(\leq 0.1)$ is interpreted to be the result of small coupling between modes of the planar (quinoidal) $S_{1}$ state and the essentially single-bonded (interring bond) twisted $\mathrm{S}_{0}$ state.

7. The decrease in $k_{\mathrm{ISC}}$ as one proceeds from $\alpha 2$ to $\alpha 7$ (with plateauing at/near $\alpha 5$, is believed to be the result of the decrease in magnitude of matrix elements of the type

$$
\left\langle{ }^{1} \Psi_{\mathrm{CT}^{\prime}}\left|\mathrm{H}^{\prime}\right|^{3} \Psi_{i}\right\rangle
$$

which may be caused by a decrease in the overlap of the electron donor-electron acceptor orbitals. In any event, the classical heavy atom effect alone is not able to explain this observation. 
8. The increase in $\phi_{F}$ with increasing $n(\alpha 2-\alpha 7)$ arises primarily because of a decrease in $k_{\text {isc }}$ (see item 7 above) and not from an increase in $k_{\mathrm{F}}^{0}\left(k_{\mathrm{F}}^{0}\right.$ is essentially constant over all $\left.\alpha n^{\prime} s\right)$.

9. "No" phosphorescence has been abserved by us or others $^{26-28}$ for $\alpha 2-\alpha 4$, although the $\phi_{\mathrm{T}}$ is very high $(0.70-$ 1.0). This is believed to be the result of a large radiationless rate constant.

10. There is a dramatic decrease in $\tau_{\mathrm{T}}$ from $\alpha 2$ to $\alpha 7$ ( $\geq 100$ to $20 \mu \mathrm{s})$. This is interpreted based on the premise that the lifetimes represent essentially the radiationless ones. The change in $\tau_{\mathrm{T}}$ would then be expected, since the lifetimes should decrease as the $T_{1}-S_{0}$ energy gap decreases because of a signficant increase in the Franck-Condon factors between $T_{1}$ and $S_{0}$ upon which the radiationless rate depends.

11. All $\phi_{\mathrm{F}}, \phi_{\mathrm{F}}, k_{\mathrm{isc}}$, and $\tau_{\mathrm{T}}$ reach a plateau at or near $\alpha 5$. These results, coupled with some absorption and fluorescence data, are interpreted as indicating that $\alpha 5$ acts as the first representative of an " $\alpha$-polythiophene".

12. Triplet $\alpha$ 's can transfer energy to ${ }^{3} \mathrm{O}_{2}$ to produce ${ }^{1} \mathrm{O}_{2}$ with high efficiency where the $\phi_{\Delta}$ values are essentially the same as $\phi_{\mathrm{T}}$ for $\alpha 2, \alpha 4$, and $\alpha 5$ and only $15 \%$ lower than $\phi_{\mathrm{T}}$ for $\alpha 3$. It appears that the efficiency for $\alpha 6$ and $\alpha 7$ is less, which could be caused by the low energy of the triplets (and possibly also steric factors for the dibutyl-substituted $\alpha 7$ ).

13. Up to 10 different methods of calculations have been used to explore the most stable geometric forms of $\alpha 2-\alpha 5$ (twisting $\mathrm{S}-\mathrm{C}-\mathrm{C}-\mathrm{S}$ angles) and the $\Delta H$ between the all-trans and all-cis forms. These include three levels of $a b$ initio. In general MOPAC and MNDO93,PM3 predicted a most stable geometry of all-cis that disagreed with all other calculation methods that predicted all-trans (the former of which is certainly incorrect). For $\alpha 2-\alpha 5$, the all-trans conformer is predicted to be the most stable from among all possible conformers ( 2 for $\alpha 2,3$ for $\alpha 3$, and 10 for $\alpha 5)$. For $\alpha 3$, MOPAC,MM2, DGauss, and $a b$ initio STO3G indicate a near planar structure for the tt conformer. For $\alpha 5$, ab initio STO3G alone indicates a near $\mathrm{t}$ planar structure for the $\mathrm{ttt}$ conformer.

Acknowledgment. Part of this work was supported by JNICT-Portugal (STRD/C/AMB/49792) and by the Italian Ministero per l'Universitá e la Ricerca Scientifica e Tecnologica (MURST) and Consiglio Nazionale delle Ricerche (CNR). Drs. J. Kagan, H. Naarmann, and H. Winberg are again acknowledged for providing samples of $\alpha 4, \alpha 6$, and $\alpha 7$, respectively. J.S.M. thanks Dr. I. Conçalves for experimental support in the synthesis of $\alpha 5$. We thank Dr. Paritosh Das, Phillips Petroleum Co., for carrying out several of the higher level calculations.

\section{References and Notes}

(1) Becker, R. S.; Seixas de Melo, J.; Maçanita, A. L.; Elisei, F. Pure Appl. Chem. 1995, 67, 9.

(2) Chosrovian, H.; Rentsch, S.; Grebner, D.; Dahn, D. U.; Birchner, E. Synth. Met. 1993, 60, 23.

(3) Colditz, R.; Grebner, D.; Hebig, M.; Reutsch, S. Chem. Phys. 1995, $201,309$.

(4) Oelkrug, D.; Egelhaaf, H.-I.; Wilkinson, F. J. Fluoresc., in press. 165.

(5) Birnbaum, D.; Fichou, D.; Kohler, B. E. J. Chem. Phys. 1992, 96,

(6) Periasamy, N.; Daneli, R.; Ruani, G.; Zamboni, R.; Talani, C. Phys. Rev. Lett. 1992, 68, 919.

(7) Birnbaum, D.; Kohler, B. E. J. Chem. Phys. 1992, 96, 2492. 8819 .

(8) Belijonne, D.; Shuai, Z.; Bredas, J. L. J. Chem. Phys. 1993, 98 ,

(9) Negri, F.; Zgierski, M. Z. J. Chem. Phys. 1994, 100, 2571.

(10) Soos, Z. B.; Galvão, D. S. J. Phys. Chem. 1994, 98, 1029.

(11) Rubio, M.; Merchán M.; Orti, E.; Roos, B. Personal communication.

(12) Van Hecke, G. R., Horrocks, De W., Jr. Inorg. Chem. 1966, 5, 1968.]
(13) Seixas de Melo, J.; Becker, R. S.; Maçanita, A. L. J. Phys. Chem. 1994, 98, 6054 .

(14) Strickler, S. J.; Berg, R. A. J. Chem. Phys. 1962, 37, 814.

(15) Förster, Th. Fluoreszenz Organischer Verbindungen; Vandenhoeck und Ruprecht: Göttingen, Germany, 1951.

(16) Maçanita, A. L.; Costa, F. P.; Costa, S. M.; Melo, E. C.; Santos, H. J. Phys. Chem. 1989, 93, 336.

(17) Stricker, G. In Effective Implementation of Modulation Functions in Deconvolution and Reconvolution of Analytical Signals; Bouchy, M., Ed.; University Press: Nancy, France, 1982.

(18) Elisei, F.; Aloisi, G. G.; Görner, H. J. Phys. Chem. 1991, 95, 4225.

(19) Aloisi, G. G.; Elisei, F.; Latterini, L. J. Chem. Soc., Faraday Trans. 1992, 88, 2139.

(20) Dawe, E. A.; Long, D. A.; Land, E. J. J. Chem. Soc., Faraday Trans. 1, 1977, 73, 1319.

(21) Charmichael, I.; Hug, G. L. J. Phys. Chem. Ref. Data 1986, 15, 1.

(22) Murov, S.; Charmichael, I.; Hug, G. L. Handbook of Photochemistry; M. Dekker Inc.: New York, 1993.

(23) Medinger, T.; Wilkinson, F. Trans. Faraday Soc. 1965, 61, 620 Horrocks, A. R.; Wilkinson, F. Proc. R. Soc., Ser. A 1968, 306, 257.

(24) Hurley, J. K.; Sinai, N.; Linschitz, H. Photochem. Photobiol. 1983, 38,9 .

(25) Wilkinson, F.; Helman, W. P.; Ross, A. B. J. Phys. Chem. Ref. Data 1993, 22, 113.

(26) Reyftman, J. P.; Kagan, J.; Santus, R.; Morliere, P. Photochem. Photobiol. 1985, 41,1 .

(27) Janssen, R. A.; Smilowitz, L.; Sariciftci, N. S.; Moses, D. J. Chem. Phys. 1994, 101, 1787.

(28) Evans, C.; Weir, D.; Scaiano, J. C.; MacEachern, A.; Arnason, J. T.; Morand, P.; Hallebone, B.; Leitch, L. C.; Philogene, J. R. Photochem. Photobiol. 1986, 44, 441.

(29) Xu, B.; Holdcroft, S. J. Am. Chem. Soc. 1993, 115, 8447.

(30) Scaiano, J. C.; Redmond, R. W.; Mehta, B.; Arnason, J. T. Photochem. Photobiol. 1990, 52, 655.

(31) Van Veen, E. H. Chem. Phys. Lett. 1976, 41, 535.

(32) Flicker, W. M.; Mosher, O. R.; Kuppermann, A. Chem. Phys. Lett. 1976, 38, 489 .

(33) Garcia, P.; Pernaut, P.; Hopiat, P.; Wintgens, V.; Valat, P.; Garnier, F.; Delabouglise, D. J. Phys. Chem. 1993, 97, 513.

(34) Rossi, R.; Carpita, A.; Ciofalo, M.; Houben, J. L. Gazz. Chim. Ital. 1990, $120,793$.

(35) Rossi, R.; Ciofalo, M.; Carpita, A.; Ponterini, G. J. Photochem. Photobiol., A 1993, 70, 59.

(36) Charra, C.; Fichou, D.; Nunzi, J. M.; Pfeffer, N. Chem. Phys. Lett. 1992, $192,566$.

(37) Buma, W. J.; Kohler, B. E.; Shaler, T. A. J. Phys. Chem. 1994, 98, 4990 .

(38) Visser, G. J.; Heeres, G. J.; Wolters, J.; Vos, A. Acta Crystallogr. B. 1968, 24, 467 .

(39) Samdal, S.; Samuelson, E. J.; Volden, H. Synth. Met. 1993, 59 , 259.

(40) Bucci, P.; Longeri, M.; Veracini, C. A.; Lunazzi, L. J. Am. Chem. Soc. 1974, 96, 1305.

(41) Chadwick, J. E.; Kohler, B. E. J. Phys. Chem. 1994, 98, 3631.

(42) Takayanagi, M.; Gejo, T.; Hanazaki, F. J. Phys. Chem. 1994, 98 ,

12893. Hanazaki, F. Personal communication.

(43) Van Bolhuis, F.; Wynberg, H.; Havinga, E. E.; Meijer, E. W.; Staring, E. G. I. Synth. Met. 1989, 30, 381.

(44) De Witt, L.; Blanchard, G. J.; Le Goff, E.; Benz, M. E.; Liao, J. H.; Kanatzidis, M. G. J. Am. Chem. Soc. 1993, 115, 12158. Blanchard, G. J. Personal communication.

(45) Taliani, C.; Danieli, R.; Lazzaroni, R.; Periasamy, N.; Ruani, G.; Zamboni, R. Synth. Met. 1993, 55-57, 4714.

(46) Andres, L. S.; Merchan, M.; Fulscher, M.; Roos, B. O. Chem. Phys. Lett. 1993, 211, 125.

(47) Becker, R. S. Theory and Interpretation of Fluorescence and Phosphorescence; Wiley-Interscience: New York, 1969.

(48) Foote, C. S. Light Activated Pesticides; Heitz, J. R., Downum, K. R., Eds.; ACS Symposium Series 339; American Chemical Society: Washington, DC, 1987; p 36.

(49) Ciofalo, M.; Ponterni, G. J. Photochem. Photobiol., A 1994, 83, 1.

(50) D'Auria, M.; D'Onofrio, F. Gazz. Chim. Ital. 1988, 118, 633.

(51) Egelhaaf, H.-I.; Oelkrug, D. SPIE, in press.

(52) Nunzi, J. M.; Pfeffer, N.; Charra, F.; Fichou, D. Chem. Phys. Lett., in press.

(53) Cheng, X.; Ichimura, K.; Fichou, D.; Kobayashi, T. Chem. Phys. Lett. 1991, 185, 286.

JP960852E 